\title{
Buckling folds of a single layer embedded in matrix - Theoretical solutions and characteristics
}

\author{
F.S. Jeng*, K.P. Huang \\ Department of Civil Engineering, National Taiwan University, No. 1, Sec. 4, Roosevelt Road, Taipei 10617, Taiwan
}

Received 11 May 2007; received in revised form 12 January 2008; accepted 15 January 2008

Available online 25 January 2008

\begin{abstract}
In addition to the conventionally known buckling fold, which has a basic fold-form comprising of single frequency and non-decaying amplitudes, folds with other fold-forms having dual frequencies or decaying amplitudes also exist in nature. This research explores whether these three types of buckling folds, which have been found in elastic material, still exist for visco-elastic or pure viscous materials. Analytical solutions of the fold-forms for visco-elastic and pure viscous materials are accordingly proposed. The theoretical solutions were then examined by conditions including extremely fast or slow strain rates. The results reveal the following physical characteristics of buckling fold. (1) For the studied materials, all three types of fold-forms are possible; (2) although pure viscous material itself is strain rate dependent, a strain rate does not influence the fold-form for a viscous layer embedded in viscous matrix; (3) given the same competence contrast, both the elastic and the viscous materials have the same wavelength versus the lateral shortening relationship, and (4) two measures $\left(I_{V}\right.$ and $\left.I_{E}\right)$ representing the effect of strain rate on folding, characterized with a range from 0 to 1 , are proposed for strain rate dependent cases.
\end{abstract}

(C) 2008 Elsevier Ltd. All rights reserved.

Keywords: Theoretical solutions; Decaying amplitude; Dual frequencies; Visco-elastic; Single competent layer; Strain rate index

\section{Background}

Folding of rock strata has been found to be related to the buckling of competent layer(s) within matrix for pure elastic or pure viscous materials (Karman and Biot, 1940; Biot, 1961; Currie et al., 1962; Jeng et al., 2001). Field observations have focused on the wavelength and the thickness of a competent layer (Sherwin and Chapple, 1968; Donath and Parker, 1964). The understanding and interpretation of folds have been discussed in many publications (e.g. Biot, 1957, 1959, 1961; Ramberg, 1963, 1964; Johnson, 1970, 1977; Fletcher, 1974, 1977; Hudleston, 1973; Cobbold, 1976, 1977; Price and Cosgrove, 1990; Smoluchowski, 1909; Abbassi and Mancktelow, 1992; Johnson and Fletcher, 1994; Mühlhaus et al., 1994, 1998; Hunt et al., 1996a,b; Zhang et al., 1996,

\footnotetext{
* Corresponding author. Tel./fax: +886 223645734.

E-mail address: fsjeng@ntu.edu.tw (F.S. Jeng).
}

2000; Mancktelow, 1999; Mancktelow and Abbassi, 1992; Schmalholz and Podladchikov, 1999, 2000, 2001a-c; Jeng et al., 2002). It has been found that the wavelength of buckling folds developed during the layer shortening is related to the competence contrast, which dominates the interaction between the competent layer and the surrounding matrix. Depending on the temperature and the stress during folding, both the layer and the matrix may exhibit elastic, visco-elastic, or viscous behaviors. Table 1 summarizes some existing studies for various types of materials. Plasticity of rocks can be involved in folding at shallow depths, but is not yet considered in this research.

It was revealed that both the competent layer and the matrix tend to show viscous behavior at great depth, such as sites proximal to the upper mantle where pressure and temperature conditions are extremely high. With decreasing depth, the strata exhibit more elastic behavior as a result of reduction in pressure and temperature. Furthermore, under similar temperature and pressure conditions, the matrix exhibits more viscous behavior than the layer does; conversely, the layer may 


\begin{tabular}{|c|c|c|c|}
\hline \multicolumn{2}{|c|}{ Nomenclature } & $m_{3}$ & constant; \\
\hline A & coefficient of waveform, indicating the magnitude & $p$ & hydrostatic pressure; \\
\hline & of amplitude; & $q$ & load applied by the matrix in $y$-direction; \\
\hline $\begin{array}{l}D_{e} \\
E\end{array}$ & $\begin{array}{l}\text { Deborah number; } \\
\text { elastic Young's modulus of the layer; }\end{array}$ & $r$ & $\begin{array}{l}\text { parameter relating to amplitude of fold (Biot, } \\
\text { 1961); }\end{array}$ \\
\hline$E_{o}$ & elastic Young's modulus of the matrix; & $R$ & competence contrast \\
\hline $\bar{E}$ & $\begin{array}{l}\text { elastic Young's modulus of the layer (plane strain } \\
\text { condition); } \bar{E}=E / 1-\nu^{2}\end{array}$ & $\begin{array}{l}R_{E} \\
\bar{R}_{E}\end{array}$ & $\begin{array}{l}\text { elastic competence contrast; } \\
\text { elastic competent contrast (plane strain condition, }\end{array}$ \\
\hline $\bar{E}_{o}$ & $\begin{array}{l}\text { elastic Young's modulus of the matrix (plane strain } \\
\text { condition); } \bar{E}_{o}=E_{o} / 1-\nu_{o}^{2}\end{array}$ & $R_{V}$ & $\begin{array}{l}\left.\bar{R}_{E}=\bar{G} / G_{o}\right) \\
\text { viscous competence contrast; }\end{array}$ \\
\hline$E$ & $\begin{array}{l}\text { equivalent Young's modulus of the layer; } E= \\
3 / 2 \bar{E} \text {; }\end{array}$ & $T$ & $\begin{array}{l}\text { time elapsed from the beginning of lateral shorten- } \\
\text { ing until the initiation of buckling; }\end{array}$ \\
\hline$f$ & compressive stress in $x$-direction applied on layer; & $T_{\mathrm{R}}$ & relaxation time of the layer; \\
\hline$f_{\text {cr }}$ & $\begin{array}{l}\text { critical compressive stress in } x \text {-direction applied on } \\
\text { layer; }\end{array}$ & $\begin{array}{l}T_{\mathrm{R} o} \\
\left(T_{\mathrm{R}}\right)_{E-V}\end{array}$ & $\begin{array}{l}\text { relaxation time of the matrix; } \\
\text { general relaxation time for an elastic layer in vis- }\end{array}$ \\
\hline$G$ & elastic shear modulus of the layer; & & cous matrix; $\left(T_{\mathrm{R}}\right)_{E-V}=\eta_{o} / \bar{G}$ \\
\hline$\underline{G_{o}}$ & elastic shear modulus of the matrix; & $Y(x, t)$ & waveform function of the fold; \\
\hline $\bar{G}$ & $\begin{array}{l}\text { elastic shear modulus of the layer (plane strain con- } \\
\text { dition); } \bar{G}=G / 1-\nu\end{array}$ & $\alpha$ & $\begin{array}{l}\text { the characteristic value of the characteristic } \\
\text { equation; }\end{array}$ \\
\hline $\bar{G}_{o}$ & $\begin{array}{l}\text { elastic shear modulus of the matrix (plane strain } \\
\text { condition); } \bar{G}_{o}=G_{o} / 1-\nu_{o}\end{array}$ & $\begin{array}{l}\varepsilon \\
\varepsilon_{x}\end{array}$ & $\begin{array}{l}\text { strain distributed in layer induced by deflection; } \\
\text { strain in } x \text {-direction; lateral strain; }\end{array}$ \\
\hline$h$ & thickness of the layer; & $\varepsilon_{y}$ & strain in $y$-direction; \\
\hline$I$ & inertial moment of the layer; & $\varepsilon_{\mathrm{Z}}$ & strain in $z$-direction; \\
\hline$I_{E}$ & $\begin{array}{l}\text { strain rate index corresponding to elasticity; }\left(I_{E}=\right. \\
\left.1-I_{V}\right)\end{array}$ & $\begin{array}{l}\varepsilon_{x}^{B} \\
\varepsilon_{x}^{\mathrm{cr}}\end{array}$ & $\begin{array}{l}\text { the } \varepsilon_{x} \text { at which buckling is initiated; } \\
\text { the } \varepsilon_{x}^{B} \text { at critical (dominant) state; }\end{array}$ \\
\hline$I_{V}$ & strain rate index corresponding to viscosity; & $\dot{\varepsilon}_{x}$ & strain rate in $x$-direction; \\
\hline$k$ & spring constant, Hook’s constant; & $\dot{\varepsilon}_{y}$ & strain rate in $y$-direction; \\
\hline$L$ & wavelength; & $\dot{\varepsilon}_{z}$ & strain rate in $z$-direction; \\
\hline$l$ & normalized wavelength; $l=L / h$ & $\delta$ & the lateral ( $x$-direction) shortening length of the \\
\hline$l_{1}$ & $\begin{array}{l}\text { the } 2 \text { nd normalized wavelength of a dual frequen- } \\
\text { cies fold-form; }\end{array}$ & $\eta$ & $\begin{array}{l}\text { layer; } \\
\text { viscosity of the competent layer; }\end{array}$ \\
\hline$l_{2}$ & $\begin{array}{l}\text { the } 1 \text { st normalized wavelength of a dual frequen- } \\
\text { cies fold-form; }\end{array}$ & $\begin{array}{l}\eta_{o} \\
\lambda\end{array}$ & $\begin{array}{l}\text { viscosity of the matrix; } \\
\text { frequency of the waveform; }=2 \pi / L \text {; }\end{array}$ \\
\hline & the normalized wavelength of decaying fold-forms; & $\lambda_{\mathrm{d}}$ & the dominant wavelength ratio; \\
\hline$l_{3, \max }$ & $\begin{array}{l}\text { the maximum normalized wavelength of decaying } \\
\text { fold-forms; }\end{array}$ & $\begin{array}{l}\sigma_{i j}^{\prime} \\
\sigma_{x}\end{array}$ & $\begin{array}{l}\text { the deviatoric stress tensor; } \\
\text { normal stress in } x \text {-direction; }\end{array}$ \\
\hline$L_{\mathrm{d}}$ & dominant wavelength; & $\sigma_{y}$ & normal stress in $y$-direction; \\
\hline$l_{\mathrm{d}}$ & normalized dominant wavelength $\left(L_{\mathrm{d}} / h\right)$; & $\sigma_{z}$ & normal stress in $z$-direction; \\
\hline & the moment induced within the layer; & $\nu$ & Poisson's ratio of the layer; \\
\hline$m$ & the value of strain rate $\dot{\varepsilon}_{x}$ & $\nu_{o}$ & Poisson's ratio of the matrix. \\
\hline
\end{tabular}

show more elastic behavior than the matrix. The possible combinations that may occur along a profile of the structural level are shown in Fig. 1. Other combinations, such as a viscous layer in elastic matrix, are less likely to occur and are not considered in this work.

For a single competent layer embedded in the matrix, analytical solutions for elastic, viscous and visco-elastic materials have been proposed (Karman and Biot, 1940; Biot, 1957, 1959, 1961; Budd and Peletier, 2000; Cobbold, 1976, 1977; Currie et al., 1962; Fletcher, 1974, 1977; Johnson and Fletcher, 1994; Mühlhaus et al., 1998; Price and Cosgrove, 1990; Ramberg, 1963, 1964). Further study reveals that, given the right constitutive relations, even fractal geometries can result (Hunt et al., 1996b). These solutions focus on foldforms with single frequency and non-decaying amplitudes, like the analytical solutions found in the 1960's (Biot, 1961; Currie et al., 1962; Fig. 1).

On the other hand, since fold-forms with dual frequencies or decaying amplitudes also occur naturally (Fig. 2, Roberts, 1989), analytical solutions for these fold-forms have been accordingly proposed for elastic material (Jeng et al., 2002). It has been revealed that fold-forms of two- or three-dimensional folding can possess more than one frequency, depending on the viscous and elastic material properties of the strata (Mühlhaus et al., 1998). As a result, three types of fold-forms are identified for the elastic layer-matrix system (as illustrated 
Table 1

Summary of some existing researches on buckle folding

\begin{tabular}{|c|c|c|}
\hline \multicolumn{2}{|c|}{ Material Model } & \multirow[t]{2}{*}{ References } \\
\hline Layer & Matrix & \\
\hline Elastic & Elastic & $\begin{array}{l}\text { Smoluchowski (1909), Karman and Biot (1940) } \\
\text { Currie et al. (1962) and Jeng et al. (2001, 2002) }\end{array}$ \\
\hline Viscous & Viscous & $\begin{array}{l}\text { Biot (1961), Ramberg (1963), Sherwin and } \\
\text { Chapple (1968), Dieterich and Carter (1969), } \\
\text { Hudleston and Stephansson (1973), Fletcher } \\
\text { (1974, 1977), Hudleston and Lan (1993), } \\
\text { Shimamoto and Hara (1976), Williams et al. } \\
\text { (1977), Smith (1977) and Mühlhaus et al. (1994 } \\
\text { 1998). }\end{array}$ \\
\hline Elastic & Viscous & Biot (1961) and Budd and Peletier (2000). \\
\hline Elastic & Visco-elastic & $\begin{array}{l}\text { Hunt et al. (1996a,b) and Whiting and Hunt } \\
\text { (1997) }\end{array}$ \\
\hline Visco-elastic & Viscous & $\begin{array}{l}\text { Schmalholz and Podladchikov (1999, 2000; } \\
\text { 2001) and Mühlhaus et al. (1998). }\end{array}$ \\
\hline Visco-elastic & Visco-elastic & $\begin{array}{l}\text { Abbassi and Mancktelow (1990, 1992), Zhang } \\
\text { et al. (1996, 2000), Mancktelow (1999), } \\
\text { Schmalholz and Podladchikov (1999, 2000, } \\
\text { 2001a-c) and Jeng et al. (2002). }\end{array}$ \\
\hline
\end{tabular}

in Fig. 3): (1) Type $A-$ a dual frequencies with non-decaying amplitude; (2) Type $B$ - single frequency with non-decaying amplitude, which is often called dominant wavelength; and (3) Type $C$ - single frequency with decaying fold-form.
For the elastic layer-matrix system, the configurations of the three basic fold-forms are found to be (Jeng et al., 2002):

Type A: $Y(x)=a_{1} \sin \left(\frac{2 \pi}{l_{1}} x\right)+b_{1} \sin \left(\frac{2 \pi}{l_{2}} x\right)$

Type B: $Y(x)=a_{2} \sin \left(\frac{2 \pi}{l_{\mathrm{d}}} x\right)$

Type $C: Y(x)=\mathrm{e}^{-m_{3} x} a_{3} \sin \left(\frac{2 \pi}{l_{3}} x\right)$

where $a_{i}$ and $b_{i}$ are constants that control the amplitude and the phase-shift of the fold and are determined by the boundary condition inducing buckling of the competent layer. The term $m_{3}$ represents the magnitude of amplitude decay. The definitions of $l_{1}, l_{2}, l_{\mathrm{d}}$ and $l_{3}$ are illustrated in Fig. 3. In addition to the theoretical solutions, numerical analyses using finite element method confirm that the three fold-forms can be formed for elastic materials (Jeng et al., 2002).

When an elastic layer is embedded in elastic matrix and its Types $A$ and $B$ fold-forms are considered, the wavelengths $l$ are related to the global, lateral shortening $\varepsilon_{x}^{B}$, which is induced by lateral stress $f$ and $f=\bar{E} \varepsilon_{x}^{B}$, as:

$\varepsilon_{x}^{B}=\frac{\pi^{2}}{3 l^{2}}+\frac{E_{o} l}{2 E \pi}$

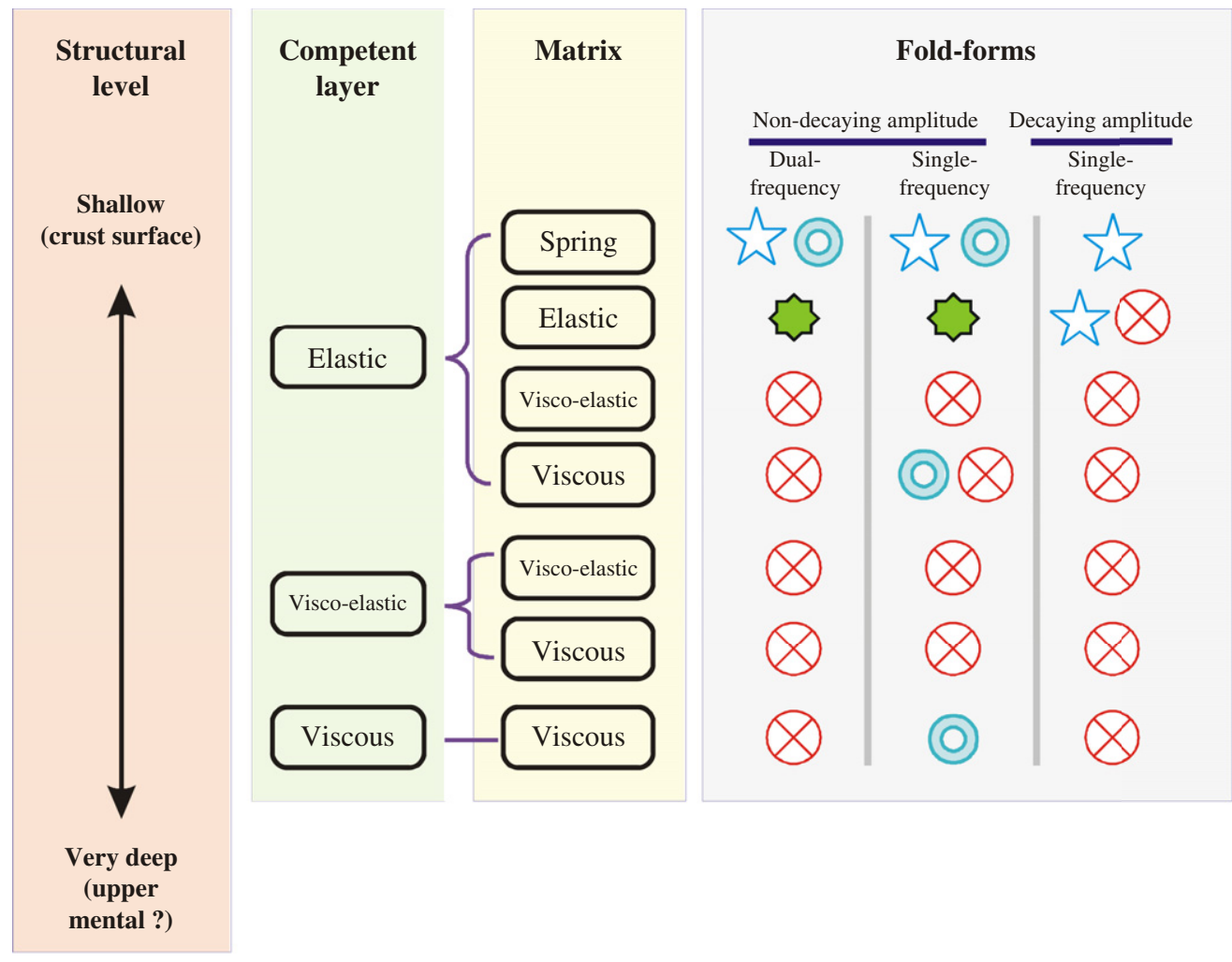

Remarks: (): Biot, 1961 ; 1 : Currie et al., 1962; $\hat{W}$ : Jeng et al., $2002 ; \bigotimes$ : This research.

Fig. 1. Various combinations of material types used by some existing solutions and the proposed solutions. 


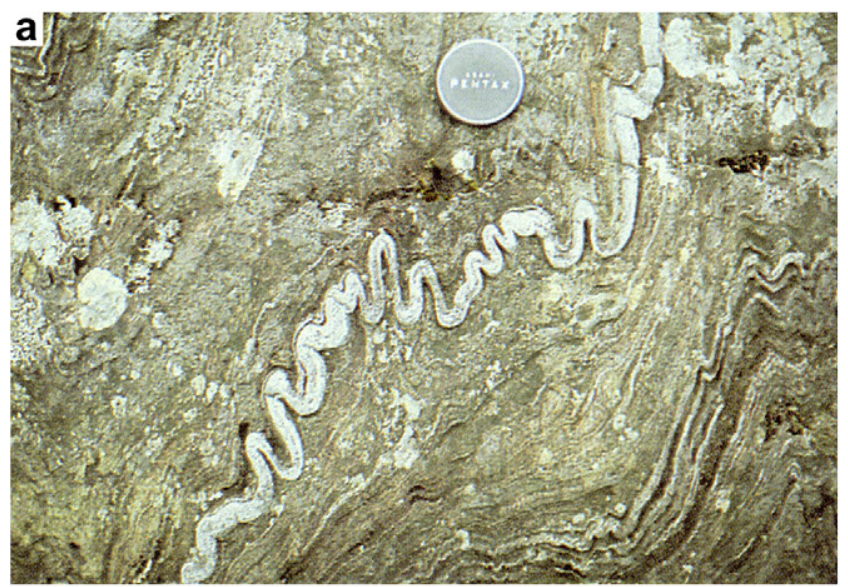

A dual-frequency fold-form

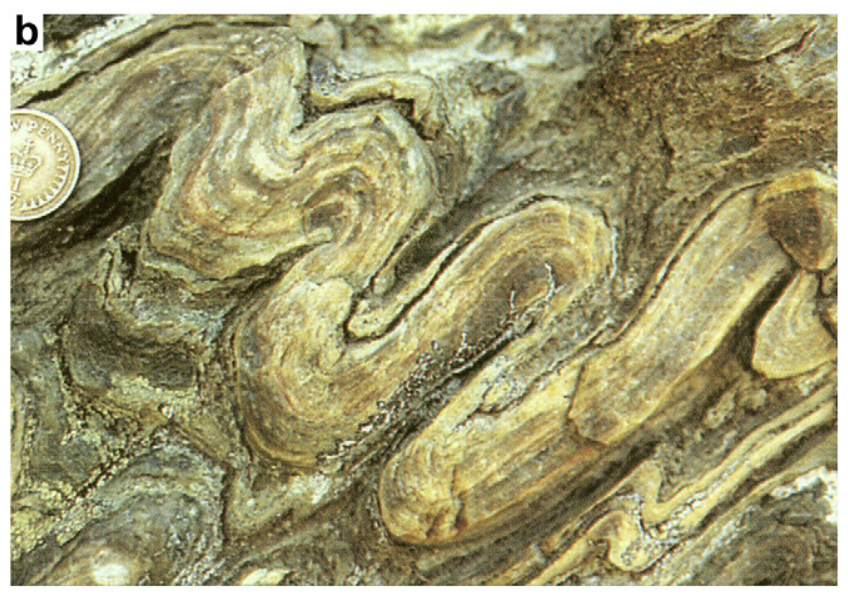

A fold-form with a decaying amplitude

Fig. 2. Natural folds with a dual frequencies and a decaying amplitude (Roberts, 1989). The fold in the upper photo exhibits dual frequencies and the one in the lower photo exhibits decaying amplitude (from the right toward the left of the photo).
Eq. (3) has been proposed by Currie et al. (1962) and is the upper part curve shown in Fig. 3. Accordingly, the dominant wavelength $l_{\mathrm{d}}$ is:

$l_{\mathrm{d}}=2 \pi \sqrt[3]{\frac{E}{6 E_{o}}}$

Based on the numerical analysis of Type $C$ fold-forms, an empirical $\varepsilon_{x}^{B}-l$ relationship was proposed as (Jeng et al., 2002):

$\varepsilon_{x}^{B}=\frac{4 \pi^{2}}{3 l^{2}}-\frac{1}{2} \sqrt[3]{\frac{E_{o}^{2}}{6 E^{2}}}$

Fig. 3 illustrates the relations of the wavelength and the lateral shortening, expressed in terms of lateral strain $\varepsilon_{x}^{B}$, when buckling occurs for the three types of fold-forms. The curves are responses of the layer-matrix system when buckle folding occurs at varying $\varepsilon_{x}^{B}$ levels and are hereinafter referred to as $\varepsilon_{x}^{B}-$ $l$ curves. For an elastic layer-matrix system, a greater $\varepsilon_{x}^{B}$, exceeding $\varepsilon_{x}^{\mathrm{cr}}$, leads to a dual frequencies fold-form. Types $B$ and $C$ fold-forms can be obtained when $\varepsilon_{x}^{B}$ equals $\varepsilon_{x}^{\mathrm{cr}}$ or is less than $\varepsilon_{x}^{\mathrm{cr}}$, respectively. The $\varepsilon_{x}^{B}-l$ curve is selected to replace the $F-$ $l$ curve adopted by Jeng et al. (2002), since the measure $\varepsilon_{x}^{B}$, in which the influence of the layer rigidity (the Young's modulus $E$ and the thickness of the layer $h$ ) has been normalized, is dimensionless and can represent more general situations.

To verify whether the relations shown in Fig. 3 exist for visco-elastic or viscous materials, the analytical solutions for Types $A, B$ and $C$ fold-forms must be studied. As such, this research aims at developing the analytical solutions for all combinations of the layer-matrix systems shown in Fig. 1.

Based on the proposed theoretical solutions listed in Table 1 , this paper further explores the mechanism of folding, focusing on the following aspects. (1) The physical characteristics revealed by these solutions; (2) the transitions of the
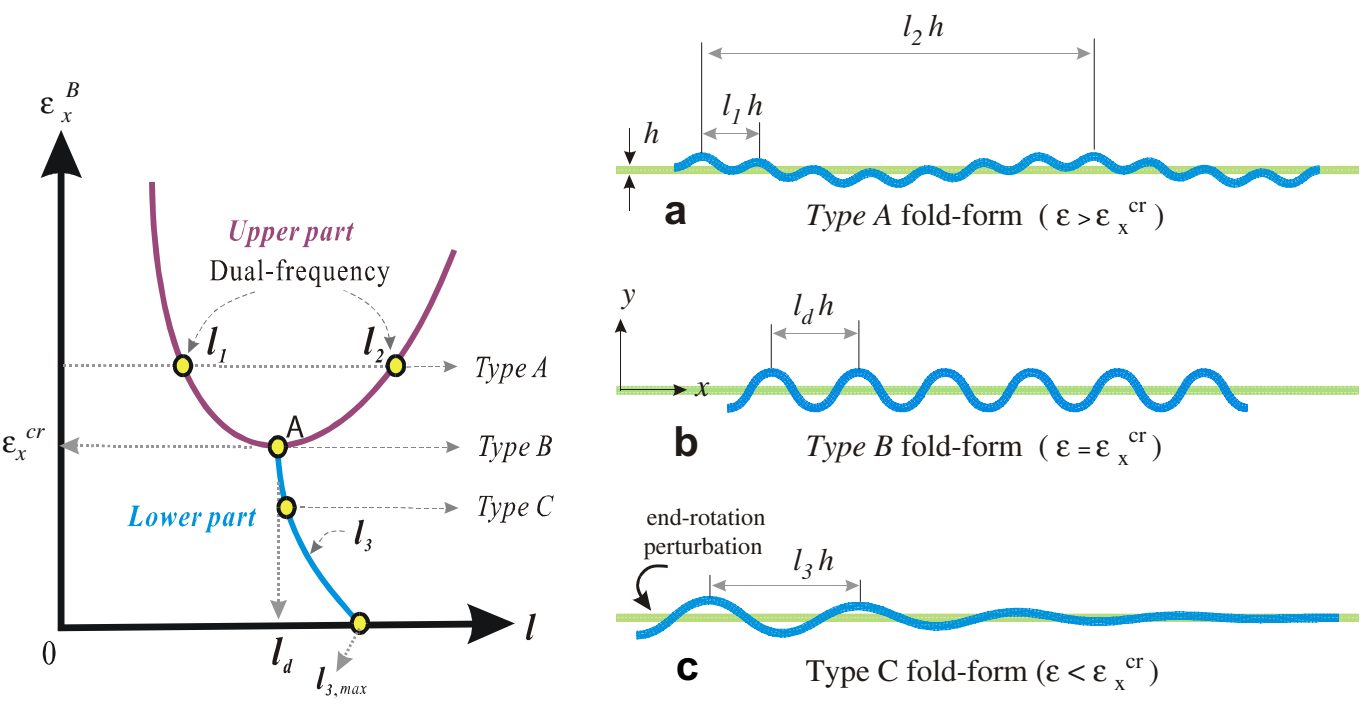

Fig. 3. Schematic illustrations of the three types of fold-forms and the $\varepsilon_{x}^{B}-l$ relationships, which have been obtained from analytical solutions and the numerical analysis. (a) Type $A$ fold-form comprises two frequencies, which can be yielded when $\varepsilon_{x}>\varepsilon_{x}^{\text {cr }}$. (b) Type $B$ fold-form is a single frequency wave with a constant amplitude over the full length of the competent layer (when $\varepsilon_{x}=\varepsilon_{x}^{\mathrm{cr}}$ ). (c) Type $C$ fold-form is a single frequency wave characterized by an amplitude attenuation away from the perturbed end (when $\varepsilon_{x}<\varepsilon_{x}^{\mathrm{cr}}$ ). 
visco-elastic material into other materials under extreme strain rates; (3) the joint influences of elastic and viscous competence contrasts; (4) the influence of strain rates; and (5) the discussion of measures of strain rates, e.g. the dominant wavelength ratio $\lambda_{\mathrm{d}}$ (Schmalholz and Podladchikov, 1999, 2000, 2001c). Two measures of strain rates are accordingly proposed in the present paper.

\section{Theoretical solutions for non-decaying fold-forms}

Two-component notations are used hereinafter to indicate the layer-matrix system. For instance, the notations $E-E$, $E-E V$ and $E-V$ represent the cases of an elastic layer embedded in the elastic, visco-elastic and viscous matrix, respectively. The definitions of symbols, provided that they are not included in the text, can be found in the attached nomenclature.

The following assumptions are made.

(1) The material models adopted are simplified and idealized. For instance, the Maxwell model is adopted for viscoelastic materials.

(2) Since the considered strain rates are slow, inertial forces are neglected.

(3) Compression is applied at both ends of the model at a constant strain rate, parallel to the competent layer.

(4) The analysis is restricted to a plane strain condition in two dimensions. At the initial stage of buckling or at the fold nucleation stage, the amplitude of the folds is small enough so that a small strain theory is applicable.

(5) Although a two-dimensional waveform is considered, the governing equation originates from one-dimensional equilibrium of the layer-matrix system; that is, the lateral interaction of stress is not completely involved in derivation.

(6) The layer and the matrix are well connected and separation will not occur.

The isotropic, linear elastic model is adopted for the elastic material. The viscous model selected for the viscous material is related to strain rate as: $\sigma=\eta \dot{\varepsilon}$, where $\sigma$ is the induced stress, $\eta$ is the viscosity and $\dot{\varepsilon}$ is the applied strain rate. The elastic material is compressible, while the viscous material is incompressible.

\subsection{The basic waveform}

The configurations of the layer and the surrounding material as well as the corresponding definitions are illustrated in Fig. 4. By observing the folding behaviors of the elastic layer-matrix system (the $E-E$ model) and taking Eq. (4) for instance, it has been found that the waveform and the wavelength can alternatively be related to the global shortening $\varepsilon_{x}^{B}$, instead of lateral stress $f$, at the moment of buckling. As such, the basic waveform can be related to $\varepsilon_{x}^{B}$ as:

$Y(x, t)=A \varepsilon_{x}^{B}(t) \sin \lambda x$

where $A$ is an arbitrary constant and $\lambda(=2 \pi / L)$ is a frequency of the waveform. If the material behavior is rate independent, e.g. it is an elastic material, then both $Y(x, t)$ and $\varepsilon_{x}^{B}(t)$ are reduced to $Y(x)$ and $\varepsilon_{x}^{B}$, respectively.

The governing equation for buckle folding of an elastic layer embedded in elastic matrix (the $E-E$ model) is found to be (Jeng et al., 2002):

$q=f h \frac{\partial^{2} Y}{\partial x^{2}}+\frac{1}{12} \bar{E} h^{3} \frac{\partial^{4} Y}{\partial x^{4}}$

After substituting Eq. (7) into Eq. (8a) and incorporating a reactive load exerted by the matrix $q=-\bar{E}_{o} \lambda Y$, the $\varepsilon_{x}^{B}-l$ relationship for the $E-E$ model is obtained as:

$\varepsilon_{x}^{B}=\frac{\pi^{2}}{3 l^{2}}+\frac{\bar{E}_{o} l}{2 \bar{E} \pi}$

where $\bar{E}=E /\left(1-\nu^{2}\right)$ and $\bar{E}_{o}=E_{o} /\left(1-\nu_{o}^{2}\right)$ are the equivalent of Young's modulus of the layer and the matrix under a plane strain condition, respectively.

The $\varepsilon_{x}^{B}-l$ relationship shown by Eq. (8b) is identical with that proposed by Currie et al. (1962). That is, the waveform expressed by Eq. (7) can serve as the fundamental waveform of buckle folding. The corresponding $\varepsilon_{x}^{B}-l$ relationship of visco-elastic or viscous materials will be further studied subsequently.

\subsection{A viscous layer in the viscous matrix (the $V-V$ model)}

If the viscous material is incompressible, the governing equation for folding of a viscous layer embedded in the viscous matrix (the $V-V$ case) has been found to be (Biot, 1961):

$q=f h \frac{\partial^{2} Y}{\partial x^{2}}+\frac{1}{3} \eta h^{3} \frac{\partial^{5} Y}{\partial t \partial x^{4}}$

$q=-4 \eta_{o} \lambda \frac{\partial Y}{\partial t}$

Since the lateral stress $f$ can be related to the lateral strain rate as $f=4 \eta \dot{\varepsilon}_{x}$, after differentiating Eq. (7) with respect to $x$ and $t$ and substituting the results for Eq. (9), the governing equation becomes:

$-4 \eta_{o} \lambda A \dot{\varepsilon}_{x} \sin \lambda x=-4 \eta \dot{\varepsilon}_{x} h A \lambda^{2} \varepsilon_{x}^{B} \sin \lambda x+\frac{1}{3} \eta h^{3} A \lambda^{4} \dot{\varepsilon}_{x} \sin \lambda x$

Further reduction of Eq. (10) leads to the $\varepsilon_{x}^{B}-l$ relationship as:

$\varepsilon_{x}^{B}=\frac{\pi^{2}}{3 l^{2}}+\frac{\eta_{o} l}{2 \eta \pi}$

where $h$ is the thickness of the layer and $l$ is a normalized, dimensionless wavelength $(=L / h)$.

By taking $\partial \varepsilon_{x}^{B} / \partial l=0$, at which $\varepsilon_{x}^{B}=\varepsilon_{x}^{\mathrm{cr}}$, the dominant wavelength is found to be $l_{\mathrm{d}}=2 \pi \sqrt[3]{\eta / 6 \eta_{o}}$, which is identical with that proposed by Biot (1961). The significance of Eq. (11) is that, although a viscous material is strain rate 
$\mathbf{a}$
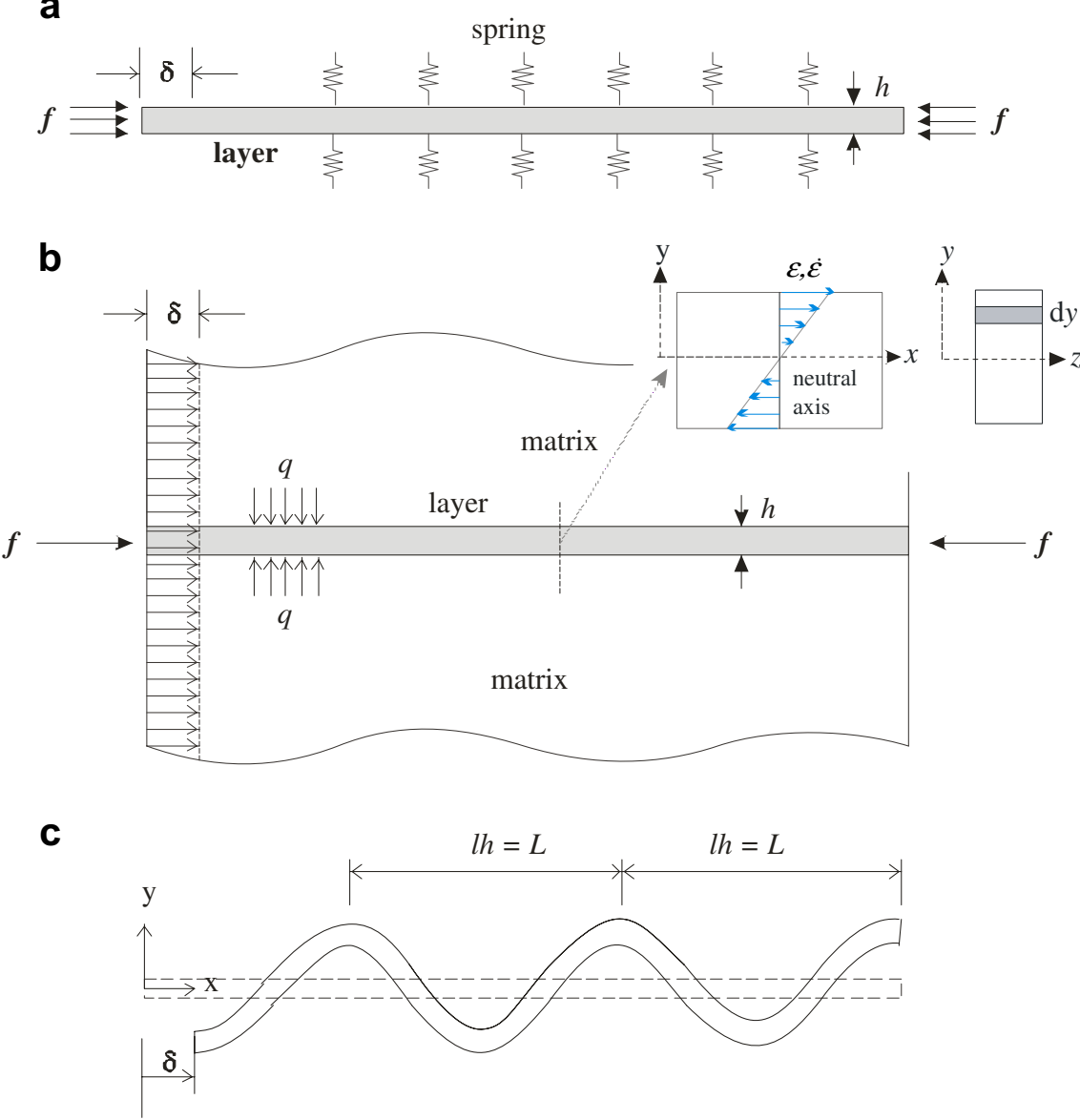

Fig. 4. Schematic illustrations for the analyzed models. (a) A layer-spring model; (b) a layer-matrix model; and (c) an illustration for the corresponding definitions.

dependent, the wavelength does not depend on the strain rate but on the accumulated lateral strain $\varepsilon_{x}^{B}$ until the moment of buckling.

\subsection{A visco-elastic layer in the visco-elastic matrix (the EV-EV model)}

The conventional Maxwell visco-elastic model, illustrated in Fig. 7, is also adopted in this research. If both elastic and viscous components of the material are incompressible, the constitutive relationship of incompressible material $(\nu=0.5)$ is:

$\dot{\varepsilon}_{i j}=\frac{\dot{\sigma}_{i j}^{\prime}}{2 G}+\frac{\sigma_{i j}^{\prime}}{2 \eta}$

where $\sigma_{i j}^{\prime}=\sigma_{i j}-p \delta_{i j}$ is the deviatoric stress tensor; the first and second terms on the right side of the equation represent the contribution of elastic and viscous components, respectively.

If the material is loaded under a constant strain rate, starting from zero initial stress and strain, the variation of stress with time can be obtained by integrating Eq. (12) as:

$\sigma_{i j}^{\prime}=2 \eta \dot{\varepsilon}_{i j}\left(1-\mathrm{e}^{-\frac{G}{\eta} t}\right)$
The constitutive relation under a constant strain rate can be found by substituting Eq. (13) for Eq. (12):

$\sigma_{i j}=p \delta_{i j}+2 \eta \dot{\varepsilon}_{i j}\left(1-\mathrm{e}^{-\frac{G}{\eta} t}\right)$

When buckling is initiated, the $\sigma_{y}$ includes two components: $\sigma_{y}$ induced by folding and $\sigma_{y}$ applied by upper and lower boundaries. Since $\sigma_{y}$ applied by boundaries will not influence the equilibrium condition of the buckling layer, this term can be taken out in later derivation. Furthermore, in a plane strain condition, $\varepsilon_{z}=\dot{\varepsilon}_{z}=0$ leads to $\sigma_{z}=p$.

These two conditions cause Eq. (14) to be:

$\sigma_{x}=4 \eta \dot{\varepsilon}_{x}\left(1-\mathrm{e}^{-\frac{G}{\eta} t}\right)$

When folding occurs, the strain rate in competent layer is related to the deflection of the layer as $\dot{\varepsilon}=-y \partial^{3} Y / \partial t \partial x^{2}$, where $y$ is defined in Fig. 4b. According to Eq. (15), the stress within the competent layer can be expressed in terms of the derivatives of $Y$ as:

$\sigma=4 \eta \dot{\varepsilon}\left(1-\mathrm{e}^{-\frac{G}{\eta} t}\right)=-4 \eta y \frac{\partial^{3} Y}{\partial t \partial x^{2}}\left(1-\mathrm{e}^{-\frac{G}{\eta} t}\right)$ 
By integrating stress along the cross-section of the layer, as shown in Fig. 4b, the induced moment within the layer are obtained as:

$M=2 \int_{0}^{\frac{h}{2}} 4 \eta \dot{\varepsilon}\left(1-\mathrm{e}^{-\frac{G_{t}}{\eta}}\right) y d y=2 \int_{0}^{\frac{h}{2}} 4 \eta y^{2} \frac{\partial^{3} Y}{\partial t \partial x^{2}}\left(1-\mathrm{e}^{-\frac{G_{t}}{\eta}}\right) \mathrm{d} y$

$M=\frac{1}{3} \eta h^{3} \frac{\partial^{3} Y}{\partial t \partial x^{2}}\left(1-\mathrm{e}^{-\frac{G}{\eta} t}\right)$

Considering the equilibrium of moments at the commencement of folding, the governing equation of the $E V-E V$ model can be found as:

$q=f h \frac{\partial^{2} Y}{\partial x^{2}}+\frac{1}{3} \eta h^{3} \frac{\partial^{5} Y}{\partial t \partial x^{4}}\left(1-\mathrm{e}^{-\frac{G}{\eta} t}\right)$

And the reactive load of the matrix is:

$q=-4 \eta_{o} \lambda \frac{\partial Y}{\partial t}\left(1-\mathrm{e}^{-\frac{G_{o}}{\eta_{o}} t}\right)$

where $f$ is the lateral compressive stress and can be expressed as $f=4 \eta \dot{\varepsilon}_{x}\left(1-\mathrm{e}^{-(G / \eta) t}\right)$.

After differentiating and substituting the basic waveform Eq. (7) for Eq. (18), the governing equation becomes:

$$
\begin{aligned}
-4 \eta_{o} \lambda A \dot{\varepsilon}_{x} \sin \lambda x\left(1-\mathrm{e}^{-\frac{G_{o}}{\eta_{o}} t}\right)= & -4 \eta \dot{\varepsilon}_{x} h A \lambda^{2} \varepsilon_{x}^{B} \sin \lambda x\left(1-\mathrm{e}^{-\frac{G}{\eta} t}\right) \\
& +\frac{1}{3} \eta h^{3} A \lambda^{4} \dot{\varepsilon}_{x} \sin \lambda x\left(1-\mathrm{e}^{-\frac{G}{\eta} t}\right)
\end{aligned}
$$

If the layer is laterally shortened under a constant strain rate, the buckle strain $\varepsilon_{x}^{B}$ is the product of strain rate $\dot{\varepsilon}_{x}$ with the elapsed time until the instance of buckling folding $T$ as $\varepsilon_{x}^{B}$ $=\dot{\varepsilon}_{x} T$. The incorporation of this condition and further reduction of Eq. (19) lead to the $\varepsilon_{x}^{B}-l$ relationship of the $E V-E V$ model as:

$\varepsilon_{x}^{B}=\frac{\pi^{2}}{3 l^{2}}+\frac{\eta_{o} l\left(1-\mathrm{e}^{-\frac{T}{T_{\mathrm{R} o}}}\right)}{2 \eta \pi\left(1-\mathrm{e}^{-\frac{T}{T_{\mathrm{R}}}}\right)}$

where $T_{R}$ and $T_{\mathrm{R} o}$ are the relaxation time, as illustrated in Fig. 7, and are defined as $T_{\mathrm{R}}=\eta / G$ and $T_{\mathrm{R} o}=\eta_{o} / G_{o}$, respectively.

Based on Eq. (20), the dominant wavelength is found to be:

$l_{\mathrm{d}}=2 \pi \sqrt[3]{\frac{\eta}{6 \eta_{o}}}\left[\frac{1-\mathrm{e}^{-\frac{T}{T_{\mathrm{R}}}}}{1-\mathrm{e}^{-\frac{T}{T_{\mathrm{R} o}}}}\right]^{1 / 3}$

The elapsed time until buckling $(T)$ is involved in Eqs. (20) and (21), indicating that the response of the $E V-E V$ model is rate dependent.
2.4. A visco-elastic layer in the viscous matrix (the EV-V model)

Similarly, the governing equation for a visco-elastic layer embedded in the viscous matrix is obtained as:

$q=f h \frac{\partial^{2} Y}{\partial x^{2}}+\frac{1}{3} \eta h^{3} \frac{\partial^{5} Y}{\partial t \partial x^{4}}\left(1-\mathrm{e}^{-\frac{G}{\eta} T}\right)$

After differentiating and substituting the basic waveform Eq. (7) for Eq. (22), the governing equation becomes:

$$
\begin{aligned}
-4 \eta_{o} \lambda A \dot{\varepsilon}_{x} \sin \lambda x= & -4 \eta \dot{\varepsilon}_{x} h A \lambda^{2} \varepsilon_{x}^{B} \sin \lambda x\left(1-\mathrm{e}^{-\frac{G}{\eta} T}\right) \\
& +\frac{1}{3} \eta h^{3} A \lambda^{4} \dot{\varepsilon}_{x} \sin \lambda x\left(1-\mathrm{e}^{-\frac{G}{\eta} T}\right)
\end{aligned}
$$

Further reduction of Eq. (23) leads to the $\varepsilon_{x}^{B}-l$ relationship of the $E V-V$ model as:

$\varepsilon_{x}^{B}=\frac{\pi^{2}}{3 l^{2}}+\frac{\eta_{o} l}{2 \eta \pi\left(1-\mathrm{e}^{-\frac{T}{T_{\mathrm{R}}}}\right)}$

Based on Eq. (24), the dominant wavelength is obtained as:

$l_{\mathrm{d}}=2 \pi \sqrt[3]{\frac{\eta}{6 \eta_{o}}}\left[1-\mathrm{e}^{-\frac{T}{T_{\mathrm{R}}}}\right]^{1 / 3}$

The elapsed time until buckling $(T)$ is involved in Eqs. (24) and (25), indicating that the response of the $E V-V$ model is rate dependent.

\subsection{An elastic layer in the visco-elastic matrix (the E-EV model)}

Similar to the previous derivation, the governing equation for an elastic layer embedded in the visco-elastic matrix can be determined as:

$q=f h \frac{\partial^{2} Y}{\partial x^{2}}+\frac{1}{12} \bar{E} h^{3} \frac{\partial^{4} Y}{\partial x^{4}}$

After differentiating and substituting the basic waveform shown in Eq. (7) for Eq. (26), the governing equation becomes:

$$
\begin{aligned}
-4 \eta_{o} \lambda A \dot{\varepsilon}_{x} \sin \lambda x\left(1-\mathrm{e}^{-\frac{G_{o}}{\eta_{o}} T}\right)= & -\bar{E} \varepsilon_{x}^{B} h A \lambda^{2} \varepsilon_{x}^{B} \sin \lambda x \\
& +\frac{1}{12} \bar{E} h^{3} A \lambda^{4} \varepsilon_{x}^{B} \sin \lambda x
\end{aligned}
$$

The relationship $\bar{E}=E /\left(1-\nu^{2}\right)=2(1+\nu) G /\left(1-\nu^{2}\right)=$ $2 G /(1-\nu)=2 \bar{G}$ is used to further reduce Eq. (27), leading to the $\varepsilon_{x}^{B}-l$ relationship of the $E-E V$ model as:

$\varepsilon_{x}^{B}=\frac{\pi^{2}}{3 l^{2}}+\frac{G_{o} l}{\bar{G} \pi} \frac{T_{\mathrm{R} o}}{T}\left(1-\mathrm{e}^{-\frac{T}{T_{\mathrm{Ro}}}}\right)$ 
Based on Eq. (28), the dominant wavelength is found to be:

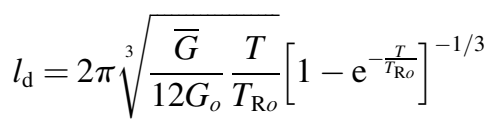

If the layer is incompressible and thus $\nu=0.5$ and $\bar{G}=2 G$, Eq. (29) becomes:

$l_{\mathrm{d}}=2 \pi \sqrt[3]{\frac{G}{6 G_{o}} \frac{T}{T_{\mathrm{Ro}}}}\left[1-\mathrm{e}^{-\frac{T}{T_{\mathrm{R} o}}}\right]^{-1 / 3}$

The elapsed time until buckling $(T)$ is involved in Eqs. (28) and (30), indicating that the response of the $E-E V$ model is strain rate dependent.

\subsection{An elastic layer in the viscous matrix (the E-V model)}

The governing equation for an elastic layer embedded in the viscous layer is obtained as:

$q=f h \frac{\partial^{2} Y}{\partial x^{2}}+\frac{1}{12} \bar{E} h^{3} \frac{\partial^{4} Y}{\partial x^{4}}$

After substituting the basic waveform in Eq. (7), the governing equation is reduced and the $\varepsilon_{x}^{B}-l$ relationship of the $E-V$ model is obtained as:

$\varepsilon_{x}^{B}=\frac{\pi^{2}}{3 l^{2}}+\frac{\eta_{o} l}{\bar{G} \pi T}$

Based on Eq. (32), the dominant wavelength is found as:

$l_{\mathrm{d}}=2 \pi \sqrt[3]{\frac{\bar{G} T}{12 \eta_{o}}}$

If the layer is incompressible and thus $\nu=0.5$ and $\bar{G}=2 G$, Eq. (33) becomes:

$l_{\mathrm{d}}=2 \pi \sqrt[3]{\frac{G T}{6 \eta_{o}}}$

The elapsed time until buckle folding $(T)$ is involved in Eqs. (32) and (34), indicating that the response of the $E-V$ model is rate dependent.

The results of the $\varepsilon_{x}^{B}-l$ relationship for the material models considered in this research and the corresponding dominant wavelengths are summarized in Table 2 .

\section{Solutions for the decaying fold-forms}

As shown in Fig. 3, the upper part, the lower part of $\varepsilon_{x}^{B}-l$ relationship and Point $A$ correspond to Types $A, C$ and $B$ foldforms, respectively. For any type of material considered, given the upper part of the $\varepsilon_{x}^{B}-l$ relationship, the lower part should connect to the upper part at its lowest point, Point A. For a model in which a layer is supported by springs only, by solving the differential equation, the lower $\varepsilon_{x}^{B}-l$ relationship (Type $C$ fold-form) can connect to the lowest point of the upper $\varepsilon_{x}^{B}-l$ relationship (Types $A$ and $B$ fold-forms), as shown in
Fig. 3. For other models, even for the $E-E$ model, the lower $\varepsilon_{x}^{B}-l$ relationship cannot connect to the lowest point of the upper part of $\varepsilon_{x}^{B}-l$ relationship. This problem is induced when transforming the differential equation from distinct support elements into continuous matrix. The Type $C$ fold-form of $E-E$ model had been found using numerical analyses and an empirical relation was proposed (Jeng et al., 2002).

When the three types of materials (elastic, elasto-viscous and viscous) are considered, a systematic method is herein developed to determine the lower $\varepsilon_{x}^{B}-l$ relationship. The need that the lower part should connect to the upper part at its lowest point, Point A, serves as a basic requirement for the $\varepsilon_{x}^{B}-l$ relationship of the Type $C$ fold-form to be developed. The following procedures demonstrate how a $\varepsilon_{x}^{B}-l$ relationship of the Type $C$ fold-form is determined and how the above-mentioned criterion is met.

\subsection{An elastic layer in the elastic matrix (the E-E model)}

When an elastic layer is supported by elastic springs, as illustrated in Fig. 4a, the governing equation for buckle folding is (Karman and Biot, 1940):

$\tilde{E} I \frac{\mathrm{d}^{4} Y}{\mathrm{~d} x^{4}}+f h \frac{\mathrm{d}^{2} Y}{\mathrm{~d} x^{2}}+2 k Y=0$

where $k$ is the Hooke's constant of spring. By incorporating the general waveform $Y(x)=\mathrm{e}^{\alpha x}$, the governing equation Eq. (35) can be obtained as (Jeng et al., 2002):

$\tilde{E} I \alpha^{4}+f h \alpha^{2}+2 k=0$

The solution of Eq. (36) is:

$\alpha= \pm \sqrt{\frac{-f h \pm \sqrt{f^{2} h^{2}-8 \tilde{E} I k}}{2 \bar{E} I}}$

The magnitudes of $f^{2} h^{2}-8 \tilde{E} I k$ determine the fold-form of the fold. The conditions of $f^{2} h^{2}-8 \tilde{E} I k>0, f^{2} h^{2}-8 \tilde{E} I k=0$ and $f^{2} h^{2}-8 \tilde{E} I k<0$ correspond to fold-forms of Types $A, B$ and $C$, respectively.

That is, when an elastic layer supported by elastic springs, the lower part of $\varepsilon_{x}^{B}-l$ relationship meets the upper part right at its lowest point. Therefore, it is strategic to make use of the upper $\varepsilon_{x}^{B}-l$ relationship to derive Type $C$ fold-form's $\varepsilon_{x}^{B}-l$ relationship for the $E-E$ model, so that the lower part will connect the upper part at its lowest point. By comparing Eq. (8a) with Eq. (35), $k$ and $\bar{E}_{o}\left(=E_{o} /\left(1-\nu_{o}^{2}\right)\right)$ can be related as:

$k=n \frac{\pi \bar{E}_{o}}{h l}$

where $n$ is a constant to be determined, and $\bar{E}_{o}$ is the equivalent of Young's modulus of the matrix under a plane strain condition.

For the critical state, since $f^{2} h^{2}-8 \tilde{E} I k=0$, we have $2 \pi / h l_{\mathrm{d}}=$ $\sqrt{f h / 2 \tilde{E} I}$ and $f_{\text {cr }}=3\left(\overline{E E}_{o}^{2} / 6\right)^{1 / 3} / 2$, hence $\tilde{E}=3 \bar{E} / 2$ is obtained. Substituting these relationships for $f^{2} h^{2}-8 \tilde{E} I k=0$, we have 


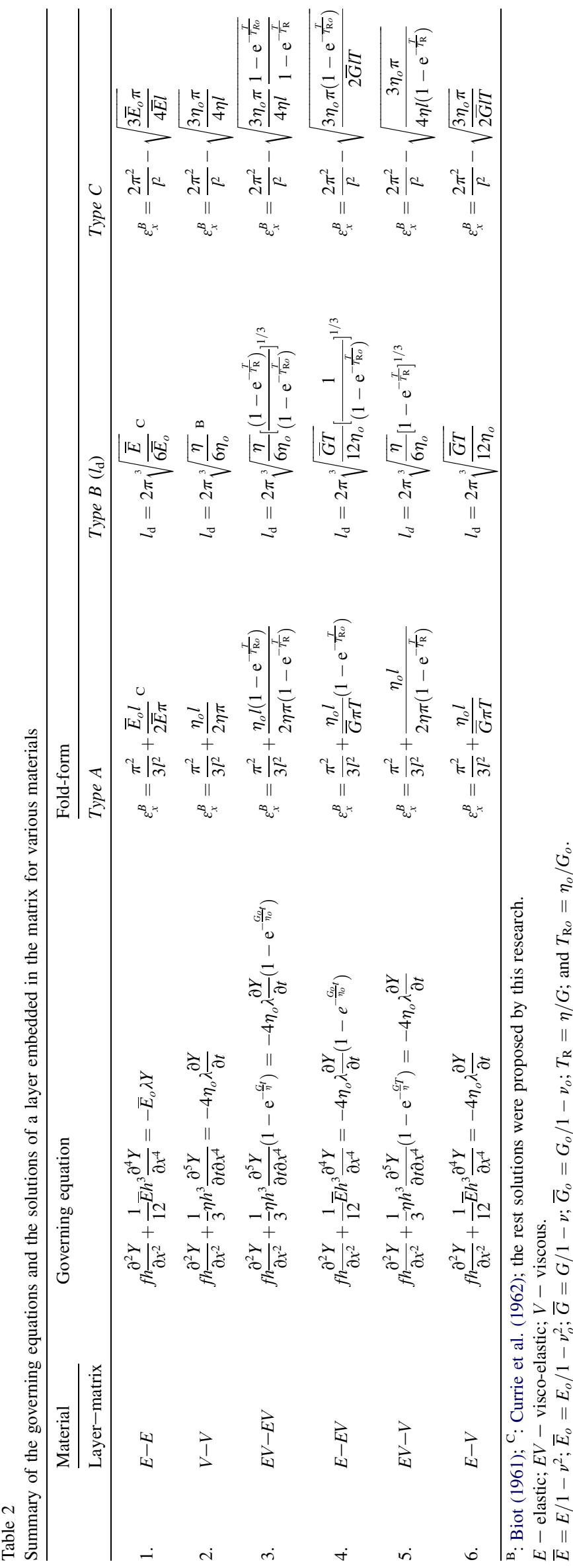

$f_{\mathrm{cr}}^{2} h^{2}=8(3 \bar{E} / 2)\left(h^{3} / 12\right) k$. As such, the value of $n$ is obtained as $n$ $=3 / 4$ and Eq. (38a) becomes:

$k=\frac{3 \pi \bar{E}_{o}}{4 h l}$

When $f^{2} h^{2}-8 \tilde{E} I k<0$, a Type $C$ fold-form appears and has the following waveform (Jeng et al., 2002):

$Y(x)=\mathrm{e}^{-m_{3} x} \sin \lambda x$

where

$m_{3}=\sqrt{\frac{-f h+\sqrt{8 \tilde{E} I k}}{4 \tilde{E} I}} ; \quad \lambda=\sqrt{\frac{f h+\sqrt{8 \tilde{E} I k}}{4 \tilde{E} I}}$

Since the magnitude of $\lambda$ is related to the frequency as $\lambda=$ $2 \pi / L$, in conjunction with the substitution of Eq. (38b) for Eq. (40), the $\varepsilon_{x}^{B}-l$ relationship for the Type $C$ fold-form is accordingly obtained as:

$\varepsilon_{x}^{B}=\frac{2 \pi^{2}}{l_{3}^{2}}-\sqrt{\frac{3 \bar{E}_{o} \pi}{4 \bar{E} l_{3}}}$

Based on Eq. (41a) and the condition that the stress $f$ is positive, the normalized wavelength is obtained as:

$l_{3}<\sqrt[3]{4} \times 2 \pi \sqrt[3]{\frac{\bar{E}}{6 \bar{E}_{o}}}=\sqrt[3]{4} l_{\mathrm{d}}$

The maximum wavelength of the Type $C$ fold-form is $l_{3, \max }=$ $\sqrt[3]{4} l_{\mathrm{d}}$, as illustrated in Fig. 3 .

\subsection{Other models}

It can be shown that $n=3 / 4$ is also valid for other materials considered, therefore using a procedure similar to the above derivation method, the $\varepsilon_{x}^{B}-l$ relationships (Type $C$ fold-form) of other models are accordingly obtained as:

(1) $V-V$ model

$\varepsilon_{x}^{B}=\frac{2 \pi^{2}}{l_{3}^{2}}-\sqrt{\frac{3 \eta_{o} \pi}{4 \eta l_{3}}}$

$l_{3}<\sqrt[3]{4} \times 2 \pi \sqrt[3]{\frac{\eta}{6 \eta_{o}}}=\sqrt[3]{4} l_{\mathrm{d}}$

(2) $E V-E V$ model

$\varepsilon_{x}^{B}=\frac{2 \pi^{2}}{l_{3}^{2}}-\sqrt{\frac{3 \eta_{o} \pi}{4 \eta l_{3}} \frac{1-\mathrm{e}^{-\frac{T}{T_{\mathrm{R} o}}}}{1-\mathrm{e}^{-\frac{T}{T_{\mathrm{R}}}}}}$

$l_{3}<\sqrt[3]{4} \times 2 \pi \sqrt[3]{\frac{\eta}{6 \eta_{o}}}\left(\frac{1-\mathrm{e}^{-\frac{T}{T_{\mathrm{R}}}}}{1-\mathrm{e}^{-\frac{T}{T_{\mathrm{R} o}}}}\right)^{1 / 3}=\sqrt[3]{4} l_{\mathrm{d}}$

(3) $E V-V$ model 
$\varepsilon_{x}^{B}=\frac{2 \pi^{2}}{l_{3}^{2}}-\sqrt{\frac{3 \eta_{o} \pi}{4 \eta l_{3}\left(1-\mathrm{e}^{-\frac{T}{T_{\mathrm{R}}}}\right)}}$

$l_{3}<\sqrt[3]{4} \times 2 \pi \sqrt[3]{\frac{\eta}{6 \eta_{o}}}\left(1-\mathrm{e}^{-\frac{T}{T_{\mathrm{R}}}}\right)^{1 / 3}=\sqrt[3]{4} l_{\mathrm{d}}$

(4) $E-E V$ model

$\varepsilon_{x}^{B}=\frac{2 \pi^{2}}{l_{3}^{2}}-\sqrt{\frac{3 \eta_{o} \pi\left(1-\mathrm{e}^{-\frac{T}{T_{\mathrm{Ro}}}}\right)}{2 \bar{G} l_{3} T}}$

$l_{3}<\sqrt[3]{4} \times 2 \pi \sqrt[3]{\frac{\bar{G} T}{12 \eta_{o}}}\left(\frac{1}{1-\mathrm{e}^{-\frac{T}{T_{\mathrm{Ro}}}}}\right)^{1 / 3}=\sqrt[3]{4} l_{\mathrm{d}}$

(5) $E-V$ model

$\varepsilon_{x}=\frac{2 \pi^{2}}{l_{3}^{2}}-\sqrt{\frac{3 \eta_{o} \pi}{2 \bar{G} l_{3} T}}$

$l_{3}<\sqrt[3]{4} \times 2 \pi \sqrt[3]{\frac{\bar{G} T}{12 \eta_{o}}}=\sqrt[3]{4} l_{\mathrm{d}}$

The solutions of the Types $A, B$ and $C$ fold-forms for the studied models are summarized in Table 2.

\section{Discussion $I$ - Strain rate independent cases (the $\mathrm{E}-\mathrm{E}$ and $\mathrm{V}-\mathrm{V}$ cases)}

When the layer-matrix system is shortened under a constant strain rate $\dot{\varepsilon}_{x}$ and the layer is buckled at the accumulated strain $\varepsilon_{x}^{B}$, the strain rate, the elapsed time until buckling $(T)$ and $\varepsilon_{x}^{B}$ can be related as: $\dot{\varepsilon}_{x} T=\varepsilon_{x}^{B}$. Therefore, the elapsed time $T$, which is the time to achieve a specific $\varepsilon_{x}^{B}$ under a constant strain rate $\dot{\varepsilon}_{x}$, can alternatively serve as a measure for strain rate. Observing the $\varepsilon_{x}^{B}-l$ relationships listed in Table 2, the $E-E$ case does not involve $T$; namely, the $E-E$ model is rate independent.

On the other hand, although the viscous material is strain rate dependent by nature, the $V-V$ case is another case not involving $T$ and is also rate independent. This indicates that, no matter how fast or slow the strain rates are, the $V-V$ case will yield an identical fold-form, as long as the strain $\varepsilon_{x}^{B}$ at buckling is the same. For strain rate independent cases, $E-E$ and $V-V$ systems, the resulting fold-forms are determined by $\varepsilon_{x}^{B}$ and the material properties ( $E$ and $E_{o}$ and, or $\eta$ and $\eta_{o}$ ).

The $\varepsilon_{x}^{B}-l$ relationships of the $E-E$ and the $V-V$ cases can be expressed in terms of the competence contrasts $\left(R_{E}\right.$ or $\left.R_{V}\right)$ as:

$\varepsilon_{x}^{B}=\frac{\pi^{2}}{3 l^{2}}+\frac{\bar{E}_{o} l}{2 \bar{E} \pi}=\frac{\pi^{2}}{3 l^{2}}+\frac{l}{2 R_{E} \pi}$

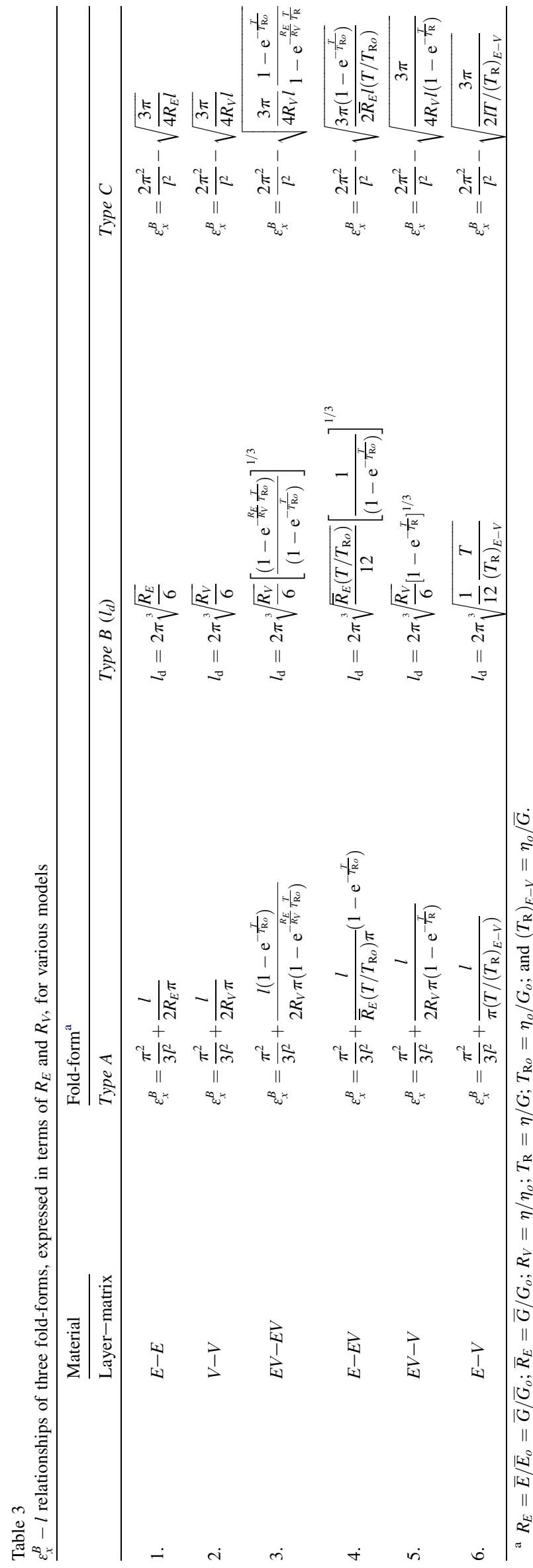


$\varepsilon_{x}^{B}=\frac{\pi^{2}}{3 l^{2}}+\frac{\eta_{o} l}{2 \eta \pi}=\frac{\pi^{2}}{3 l^{2}}+\frac{l}{2 R_{V} \pi}$

Similarly, all the solutions in Table 2 are further converted to $R_{E}$ and $R_{V}$, and the results are listed in Table 3 .

Eqs. (47a) and (47b) can be expressed in general as:

$\varepsilon_{x}^{B}=\frac{\pi^{2}}{3 l^{2}}+\frac{l}{2 R \pi}$

where $R$ is the competence contrast, either $R_{E}\left(=\bar{E} / \bar{E}_{o}\right)$ or $R_{V}\left(=\eta / \eta_{o}\right)$ for the $E-E$ and the $V-V$ cases, respectively. The $\varepsilon_{x}^{B}-l$ relationship expressed by Eq. (48) is illustrated in Fig. 5a, which involves the $\varepsilon_{x}^{B}-l$ relationship of Types $A$ and $B$ fold-forms. The first term on the right side $\left(\pi^{2} / 3 l^{2}\right)$ represents the situation when there is no matrix and is referred as the "no matrix" curve hereinafter. The second term on the right side $(l / 2 R \pi)$ is the entrance through which the influence of the matrix on the resulting wavelength

a

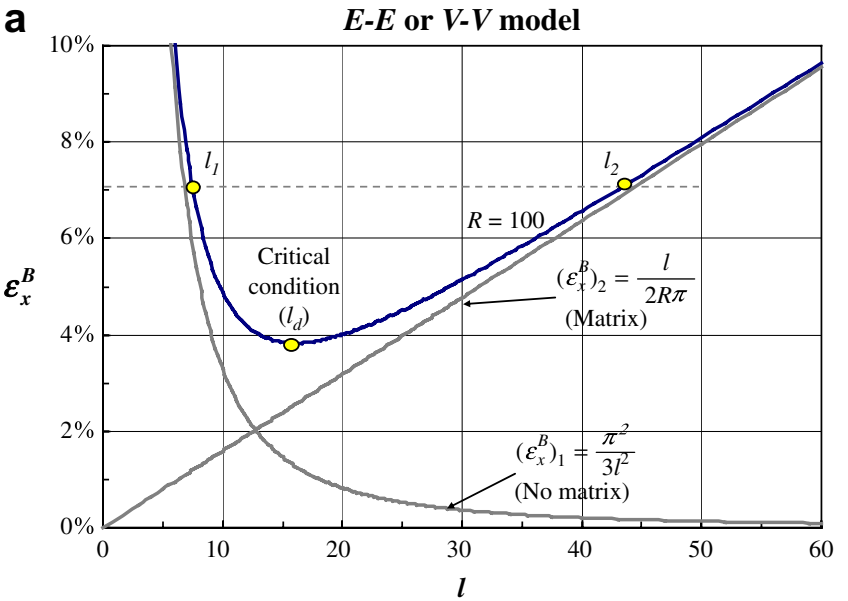

b

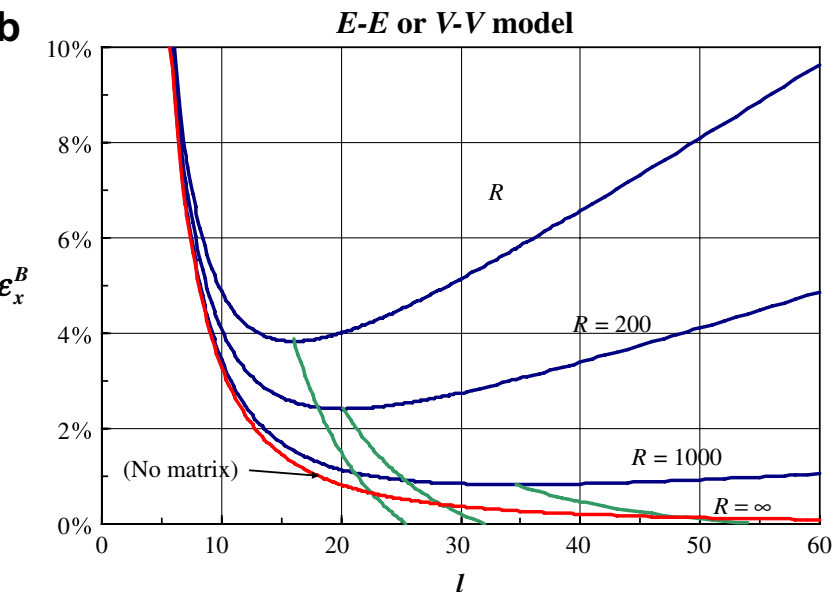

Fig. 5. The $\varepsilon_{x}^{B}-l$ relationship for either the $E-E$ model or the $V-V$ model. (a) Typical $\varepsilon_{x}^{B}-l$ relation of Types $A$ and $B$ fold-forms. The dual frequencies $\varepsilon_{x}^{B}-$ $l$ relationship comprises two parts; the first part $\pi^{2} / 3 l^{2}$ results from no matrix, and is referred as the "no matrix" curve. The second component $1 / 2 R \pi$ indicates the influence of the competent contrast. (b) Variation of the $\varepsilon_{x}^{B}-l$ curve with $R$. The range of $\varepsilon_{x}^{B}$ in which Type $C$ fold-form can occur decreases upon increasing $R$. is introduced. As illustrated in Fig. 5a, providing that $\varepsilon_{x}^{B}>$ $\varepsilon_{x}^{\mathrm{cr}}$, Type $A$ fold-form will be formed, with $l_{1}$ and $l_{2}$ mainly contributed by the "no matrix" and the "matrix" terms on the right side of Eq. (48), respectively. The wavelengths $l_{1}$ and $l_{2}$ resemble second-order and first-order folds (Price and Cosgrove, 1990) in nature.

When $\varepsilon_{x}^{B}$ is close to $\varepsilon_{x}^{\mathrm{cr}}$ (e.g., the critical case $l=l_{\mathrm{d}}$ ), influences of the two terms on the resulting wavelength are about the same, as shown in the low part curves in Fig. 5a. As depicted by Fig. 5b, an increase of $R$, or a relatively softer matrix, gives a lower $\varepsilon_{x}^{B}-l$ curve. The lower $\varepsilon_{x}^{B}-l$ curve leads to: (1) a significant increase in $l_{2} ;(2)$ an increase in $l_{\mathrm{d}}$; (3) only a minor increase in $l_{1}$; and (4) a smaller $\varepsilon_{x}^{\text {cr }}$ in order to achieve the corresponding $l_{\mathrm{d}}$. As $R$ increases, the term $l / 2 R \pi$ decreases and, after summation of the two terms at r.h.s. of Eq. (48), lowers the $\varepsilon_{x}^{B}-l$ curve.

When $R$ approaches infinity, the matrix is extremely soft, and the $\varepsilon_{x}^{B}-l$ curve approaches the "no matrix" curve, indicating that the ultimately soft matrix has no contribution to determining the fold-form. Under such circumstances, the Type $A$ fold-form no longer exists since the layer-matrix system converges to a single layer system without the matrix, in which Eq. (48) converges to $\varepsilon_{x}^{B}=\pi^{2} / 3 l^{2}$, and the fold-form cannot have dual frequencies.

For the Type $C$ fold-form, the $\varepsilon_{x}^{B}-l$ curves are illustrated in Fig. 5b. It can be seen that the range of $\varepsilon_{x}^{B}$ in which Type $C$ fold-form can occur decreases upon increasing $R$. When $R$ approaches infinity, or equivalently the "no matrix" situation, the Type $C$ fold-form cannot occur.

\section{Discussion II - The EV-EV model subjected to extreme strain rates}

It has been found that strain rate has significant impact on the folding behavior of the $E V-E V$ model (Zhang et al., 2000). For the strain rate dependent cases (the $E V-E V$, $E-E V, E V-V, E-V$ cases), it is interesting to identify the response of the layer-matrix system, when the system is subjected to extreme strain rates, i.e. very fast $(T \rightarrow 0)$ or very slow $(T \rightarrow \infty)$.

For the $E V-E V$ case, when the strain rate is extremely fast $(T \rightarrow 0)$ and with the use of L'Hôpital's theorem, Eq. (20) becomes:

$\varepsilon_{x}^{B}=\lim _{T \rightarrow 0} \frac{\pi^{2}}{3 l^{2}}+\frac{l\left(1-\mathrm{e}^{-\frac{T}{T_{\mathrm{R} o}}}\right)}{2 R_{V} \pi\left(1-\mathrm{e}^{-\frac{R_{E}}{R_{V}} \frac{T}{T_{\mathrm{R} o}}}\right)}=\frac{\pi^{2}}{3 l^{2}}+\frac{l}{2 R_{E} \pi}$

That is, the response of the $E V-E V$ system converges to the $E-E$ system upon extremely fast strain rates. This phenomenon originates from the fact that the Maxwell visco-elastic model exhibits instant elasticity.

On the other hand, if strain rate is extremely slow $(T \rightarrow \infty)$, Eq. (20) becomes: 
Table 4

Conversion of the layer-matrix system to other material models upon extreme strain rates

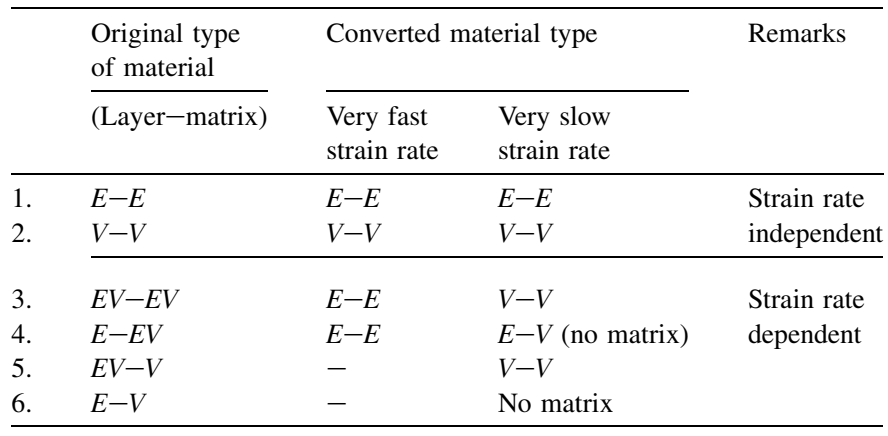

$\varepsilon_{x}^{B}=\lim _{T \rightarrow \infty} \frac{\pi^{2}}{3 l^{2}}+\frac{l\left(1-\mathrm{e}^{-\frac{T}{T_{\mathrm{Ro} o}}}\right)}{2 R_{V} \pi\left(1-\mathrm{e}^{-\frac{R_{E}}{R_{V}} \frac{T}{T_{\mathrm{Ro}}}}\right)}=\frac{\pi^{2}}{3 l^{2}}+\frac{l}{2 R_{V} \pi}$

As a result, the $E V-E V$ system converges to the $V-V$ system upon extremely slow strain rates.

Under extreme strain rates, the convergence of one layermatrix system to another system is summarized in Table 4. It indicates that the $E-E$ and the $V-V$ systems do not change since they are strain rate independent. The response of the $E-E V, E V-V$ and $E-V$ cases will be discussed in the following sections. Overall, the convergence of one system to another upon extreme strain rates reveals the physical nature of the system on one hand, and the consistency of solutions on the other.

\section{Discussion III - Factors affecting folding of strain dependent models}

\subsection{The EV-EV case}

\subsubsection{The influence of $R_{E} / R_{V}$}

As stated earlier, the $E V-E V$ case may converge to $E-E$ or $V-V$ cases upon extremely fast or extremely slow strain rates. In the case that $R_{E}=R_{V}, E-E$ and $V-V$ have the same $\varepsilon_{x}^{B}-l$ curve, by comparing Eqs. (47a) and (47b). As a result, $R_{E}=R_{V}$ induces the $E V-E V$ system to become rate independent. Such a trend can also be seen in Eq. (20); when $R_{E}=R_{V}(=R)$, Eq. (20) becomes:

$$
\varepsilon_{x}^{B}=\frac{\pi^{2}}{3 l^{2}}+\left.\frac{l\left(1-\mathrm{e}^{-\frac{T}{T_{\mathrm{R} o}}}\right)}{2 R_{V} \pi\left(1-\mathrm{e}^{-\frac{R_{E}}{R_{V}} \frac{T}{T_{\mathrm{R} o}}}\right)}\right|_{R_{E} / R_{V}=1}=\frac{\pi^{2}}{3 l^{2}}+\frac{l}{2 R \pi}
$$

However, in nature, $R_{V}$ tends to be $10-100$ times more than $R_{E}$ so that the rate independent behavior of the $E V-E V$ system is unlikely to happen in reality.

When $R_{V}>R_{E}$ and non-extreme strain rates are applied, the variation of the $\varepsilon_{x}^{B}-l$ curve with various $T / T_{\mathrm{R} o}$ is shown in Fig. 6. In Fig. 6a, the corresponding $E-E$ and the $V-V$ cases are plotted with $R_{E} / R_{V}=100 / 400\left(T_{\mathrm{R}}=4 T_{\mathrm{R}^{o}}\right)$. Since
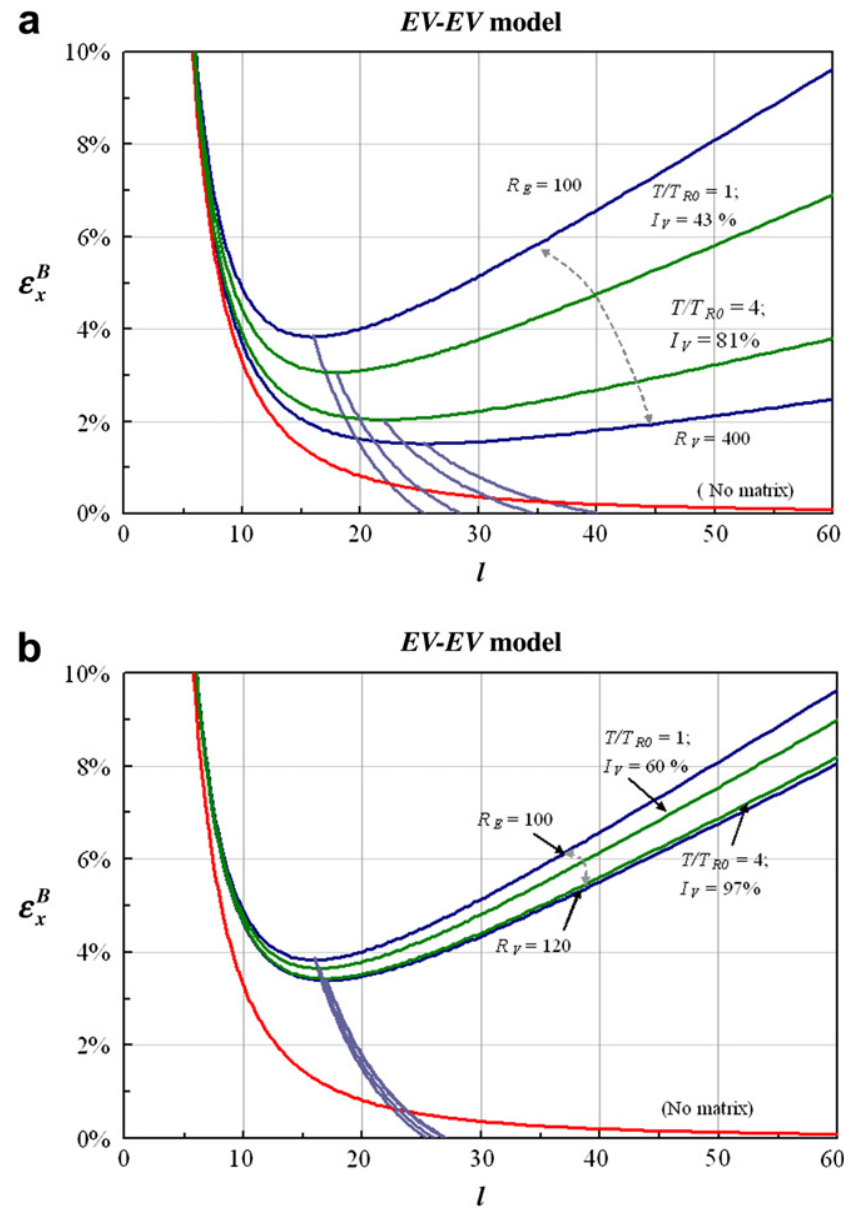

Fig. 6. The variation of the $\varepsilon_{x}^{B}-l$ curve with strain rates and influence of $R_{E} / R_{V}$ for the $E V-E V$ model. The $\varepsilon_{x}^{B}-l$ curve is bounded by the upper boundary (the elastic $\varepsilon_{x}^{B}-l$ curve, $E-E$ model) and the lower boundary (the viscous $\varepsilon_{x}^{B}-l$ curve, $V-V$ model). (a) $R_{E} / R_{V}=100 / 400$; and (b) $R_{E} / R_{V}=$ 100/120. As such, as shown in Fig. 6a, increasing $T / T_{\mathrm{Ro}}$ (represents decreasing strain rate) brings the corresponding $\varepsilon_{x}^{B}-l$ curve toward the $V-V$ curve, or the lower boundary, and vice versa.

the $E-E$ and the $V-V$ cases are extreme conditions of the $E V-E V$ system, their $\varepsilon_{x}^{B}-l$ curves serve as upper and lower boundaries of the $E V-E V$ curves enclosing the $\varepsilon_{x}^{B}-l$ curves of the $E V-E V$ system under various strain rates.

If a dimensionless term $T / T_{\mathrm{Ro}}$ in Eq. (20) is selected as a measure representing strain rate, a greater $T / T_{\mathrm{R} o}$ represents a slower strain rate. As such, as shown in Fig. 6a, increasing $T / T_{\mathrm{R} o}$ brings the corresponding $\varepsilon_{x}^{B}-l$ curve toward the $V-V$ curve, or the lower boundary, and vice versa.

If $R_{E}$ is close to $R_{V}$, e.g. $R_{E} / R_{V}=100 / 120$, the region enclosed by the upper $(E-E$ case) and the lower ( $V-V$ case) boundaries is significantly reduced, as shown in Fig. 6b. A comparison of the case shown in Fig. $6 \mathrm{~b}$ with the case shown in Fig. 6a reveals that the strain rate has relatively less influence to the resulting fold-forms when $R_{V}$ is closer to $R_{E}$. Overall, based on Fig. 6 , if $R_{E}<R_{V}$, the $\varepsilon_{x}^{B}-l$ curves of the $E-E$ model and the $V-V$ model serve as the upper and the lower boundaries of the $\varepsilon_{x}^{B}-l$ curves of the $E V-E V$ model, respectively. 


\subsubsection{The index for elastic/viscous states}

The influence of strain rate on folding has been studied by Hunt et al. (1996a), Jeng et al. (2002), Whiting and Hunt (1997), Zhang et al. (2000) and Schmalholz and Podladchikov, 1999, 2000, 2001a-c). Accordingly, measures are proposed to determine whether the applied strain rate is fast or slow enough to approach elastic or viscous conditions (Schmalholz and Podladchikov, 1999, 2000, 2001c).

The Maxwell visco-elastic material is a serial connection of an elastic component with elastic modulus $G$ and a viscous component with the viscosity $\eta$. When the Maxwell material is shortened by a constant strain rate $m$, the variation of induced stress with time is illustrated in Fig. 7. If the stress $\sigma$ is proportional to the applied strain rate $m$ and the viscosity $\eta$, the stress $\sigma$ can be normalized by $m$ and $\eta$ to be a dimensionless stress measure $\sigma / \eta m$. As shown in Fig. 7, the intersection of the initial slope of the curve (which is the elastic property of the material) with the ultimate stress $(\sigma / \eta m=1)$ corresponds to a time denoted as $T_{\mathrm{R}} . T_{\mathrm{R}}$ is conventionally called "the relaxation time". The degree at which the system approaches the pure viscosity is controlled by the elapsed time $T$, as revealed by Fig. 6. If $T$ is further normalized by the relaxation time $T_{\mathrm{R}}$ of the material, $T / T_{R}$ is a dimensionless measure, which has already excluded the influences of $m, \eta$ and $G$. If $T / T_{R}$ is less than 1 , elasticity plays a more important role in the deformational behavior as shown in Fig. 7. Alternatively, if $T / T_{R}$ is much greater than 1 , viscosity dominates the deformational behavior. Therefore, $T / T_{R}$ can serve as a fundamental measure of strain rate and can indicate the elastic or the viscous states of the material. When $T / T_{R}$ approaches infinity, the visco-elastic material reaches pure viscous state.

Although $T / T_{\mathrm{R}}$ is one of the fundamental measures of the viscous state of the material, a proper measure requires more considerations for the $E V-E V$ model. For instance, the same magnitude of $T / T_{\mathrm{R}}=4$ indicates that the material behavior is very close to a pure viscous state, as shown in Fig. 6b, whereas for another case shown in Fig. 6a, the material behavior is not so close to a pure viscous state.

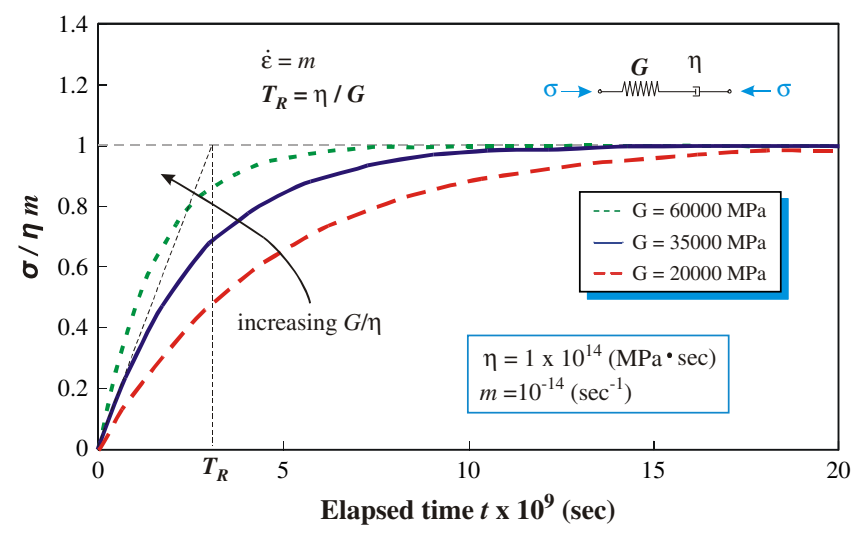

Fig. 7. The variation of stress with time when the Maxwell visco-elastic material is shortened under a constant strain rate. The induced lateral stress can be expressed as: $\sigma(t)=\eta m\left(1-\mathrm{e}^{-(G / \eta) t}\right)$. The stress is normalized by viscosity and strain rate and becomes dimensionless. $T_{R}$ is the relaxation time of the material.
In fact, existing measures, e.g., the Deborah number $D_{e}$ and the dominant wavelength ratio $\lambda_{\mathrm{d}}$ (Schmalholz and Podladchikov, 1999, 2000, 2001c), have incorporated the term $T / T_{\mathrm{R}}$ :

$D_{e}=\frac{\eta}{G} \dot{\varepsilon}=T_{\mathrm{R}} \dot{\varepsilon}=T_{\mathrm{R}} m$

$\lambda_{\mathrm{d}}=\left(\frac{\eta}{6 \eta_{o}}\right)^{1 / 3}\left(\frac{4 \eta \dot{\varepsilon}}{G}\right)^{1 / 2}=\left(\frac{R_{V}}{6}\right)^{1 / 3}\left(4 T_{\mathrm{R}} m\right)^{1 / 2}$

A comparison of Eq. (52) with Eq. (53) reveals that $\lambda_{\mathrm{d}}$ involves one more factor, the viscous competence contrast $R_{V}$, thus leading to a more reasonable representativeness of strain rate (Jeng et al., 2002).

Moreover, based on Eq. (20), a strain rate index corresponding to the viscous state $I_{V}$, is proposed as:

$I_{V}=\frac{\left(2-\mathrm{e}^{-\frac{T}{T_{\mathrm{R} o}}}-\mathrm{e}^{-\frac{R_{E}}{R_{V}} \frac{T}{T_{\mathrm{Ro}}}}\right)}{2}$

This measure is characterized by a range from 0 (pure elastic state) to 1 (pure viscous state). Meanwhile, this index is based on the folding behavior of the $E V-E V$ case, as revealed by Eq. (21), and incorporates the key terms, $T / T_{\mathrm{R} o}$ and $R_{E} / R_{V}$, since the influence of $R_{E} / R_{V}$ has been demonstrated in Fig. 6. Alternatively, a strain rate index corresponding to the elasticity $I_{E}$ is proposed as $I_{E}=1-I_{V}$.

Based on Eq. (20), the variations of the two terms, $1-\mathrm{e}^{T / T_{\mathrm{R} o}}$ and $1-\mathrm{e}^{\left(R_{E} / R_{V}\right)\left(T / T_{\mathrm{R} o}\right)}$, with $T / T_{\mathrm{R} o}$ are plotted in Fig. 8. It reveals that, for increasing $T / T_{\mathrm{R} o}$, the matrix approaches pure viscous state faster than the layer does since $1-\mathrm{e}^{T / T_{\mathrm{R} o}}$ is $1-\mathrm{e}^{\left(R_{E} / R_{V}\right)\left(T / T_{\mathrm{R} o}\right)}$ greater than $1-\mathrm{e}^{\left(R_{E} / R_{V}\right)\left(T / T_{\mathrm{R} o}\right)}$ when $R_{V}>R_{E}$. The proposed $I_{V}$ is the average of the two terms, $1-\mathrm{e}^{T / T_{\mathrm{R} o}}$ and, so that it has the significance of the "mean" viscous or elastic state of the layer and the matrix.

When the same $T / T_{\mathrm{R} o}=4$, as the two cases shown in Fig. $6 \mathrm{a}$ and b, magnitudes of $I_{V}=81 \%$ and $I_{V}=97 \%$ are helpful in indicating how close the material behavior is to the viscous state. As a result, this proposed measure is capable of

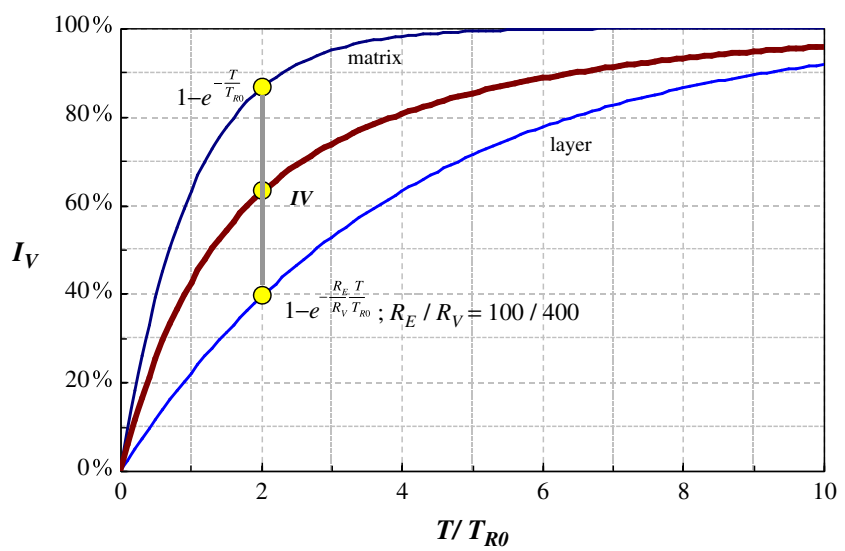

Fig. 8. The relationship of the proposed strain rate index with the viscous state of the layer and the matrix. The uppermost curve is the response of the matrix and the lowest is the response of the layer. 
distinguishing the relative viscous state for the two cases shown in Fig. 6.

Overall, when the layer-matrix system is buckled at time $T$ with the accumulated lateral strain $\varepsilon_{x}^{B}$, judgment whether the time (or the strain rate) is fast or slow, which corresponds respectively to either elastic or viscous states, can be evaluated by Eq. (54). If the assessed magnitude of $I_{V}$ is close to 1 , the material is close to the viscous state and, therefore, the applied strain rate is conceived as "slow".

\subsection{The E-EV case}

For the $E-E V$ case, the elastic layer does not have $R_{V}$ such that only $R_{E}$ and $T_{\mathrm{R} o}$ are available. Upon extremely fast strain rates $\left(T / T_{\mathrm{R} o} \rightarrow 0\right)$, the $E-E V$ model converges to the $E-E$ model, as summarized in Table 4. Consequently, the $\varepsilon_{x}^{B}-l$ curve of the $E-E$ model serves as the upper boundary of the $\varepsilon_{x}^{B}-l$ curves of the $E-E V$ model, as shown in Fig. 9.

On the other hand, when the strain rate is extremely slow $\left(T / T_{\mathrm{R} o} \rightarrow \infty\right)$, the $E-E V$ model approximates to the $E-V$ model, as shown in Table 4. In turn, when $T / T_{\mathrm{Ro}} \rightarrow \infty$, the $\varepsilon_{x}^{B}-l$ curve of $E-V$ model approximates to the "no matrix" curve, as shown in Table 4. Combining these two conditions, under ultimately slow strain rates, the $\varepsilon_{x}^{B}-l$ curve of the $E-E V$ model converges to the "no matrix" curve, as shown in Fig. 9. As a result, the "no matrix" curve serves as the lower boundary for the $\varepsilon_{x}^{B}-l$ curves of the $E-E V$ model, whereas a faster strain rate brings the $\varepsilon_{x}^{B}-l$ curve toward the upper boundary and vice versa.

In fact, the $E-E V$ model is an extreme case of the $E V-E V$ model in which the layer exhibits no viscosity. The upper boundary of the $E V-E V$ model corresponds to the $\varepsilon_{x}^{B}-l$ curve of the $E-E$ model so that the upper boundary of the $E-E$ model prevails over the $E V-E V$ model. Accordingly, the $\varepsilon_{x}^{B}-l$ curve of the $E-E$ model and the "no matrix" curve become the upper and the lower boundaries of the $E-E V$ model.

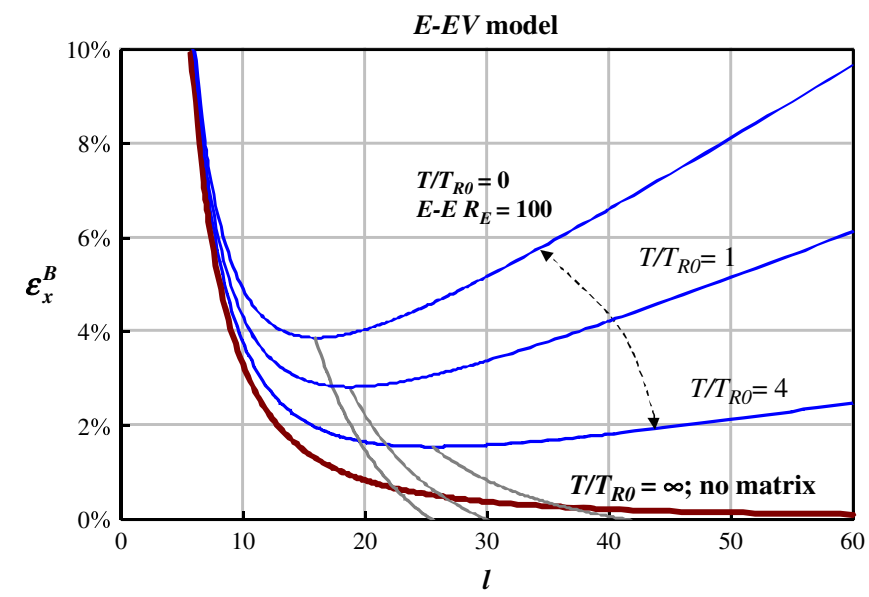

Fig. 9. The variation of the $\varepsilon_{x}^{B}-l$ curve with strain rates for the $E-E V$ model. There are upper and lower boundaries enclosed by the $\varepsilon_{x}^{B}-l$ curve. A slower strain rate brings the $\varepsilon_{x}^{B}-l$ curve closer to the lower boundary ("no matrix" curve), whereas a faster strain rate brings the $\varepsilon_{x}^{B}-l$ curve toward the upper boundary ( $E-E$ model curve).

\subsection{The EV-V case}

For the $E V-V$ case, the viscous matrix does not have $R_{E}$ such that only $R_{V}$ and $T_{\mathrm{R}}$ are available. Upon extremely slow strain rates $\left(T / T_{\mathrm{R} o} \rightarrow \infty\right)$, the $E V-V$ model converges to the $V-V$ model, as summarized in Table 4 , and follows the behavior of the $V-V$ model, namely the $\varepsilon_{x}^{B}-l$ curve. As such, the $\varepsilon_{x}^{B}-l$ curves of the $E V-V$ model have a lower boundary, which is the $\varepsilon_{x}^{B}-l$ curve of the $V-V$ model, as shown in Fig. 10. On the other hand, when strain rate is faster, the corresponding $\varepsilon_{x}^{B}-l$ curve moves upward and there is no upper boundary, as shown in Fig. 10.

The above-mentioned characteristic leads to an interesting observation: these two extreme cases of the $E V-E V$ model and the $\varepsilon_{x}^{B}-l$ curve may vary in two different regions. The regions for the $E-E V$ model and the $E V-V$ model are below or above the $\varepsilon_{x}^{B}-l$ curves of the $E-E$ model or the $V-V$ model, respectively.

\subsection{The E-V case}

For the $E-V$ model, the layer exhibits no viscosity and the matrix exhibits no elastic behavior. In this particular case, neither $R_{E}$ and $R_{V}$ are available and $T_{\mathrm{R}}$ and $T_{\mathrm{R} o}$ do not exist. Based on Eqs. (32), (33) and (46), the $E-V$ system is strain rate dependent, since $T$ exists in all equations. Therefore, a dimensionless measure of strain rate is necessary. Since the term $T / T_{\mathrm{R}}=T \eta / G$ has a physical significance of the "relaxation time", it has been adopted in other models. The term $\eta_{o} / \bar{G}$ in Eqs. (32), (33) and (46) has the unit of time and represents a general aspect of the relaxation time of the global $E-V$ system. Therefore, $\eta_{o} / \bar{G}$ is defined as $\left(T_{\mathrm{R}}\right)_{E-V}$, the relaxation time of the $E-V$ system. As such, Eqs. (32), (33) and (46) can be expressed in term of $\left(T_{\mathrm{R}}\right)_{E-V}$, as listed in Table 3 .

The variation of the $\varepsilon_{x}^{B}-l$ curve of the $E-V$ system is illustrated in Fig. 11. A slower strain rate (or a greater $T$ ) brings the $\varepsilon_{x}^{B}-l$ curves toward the "no matrix" curve. When strain

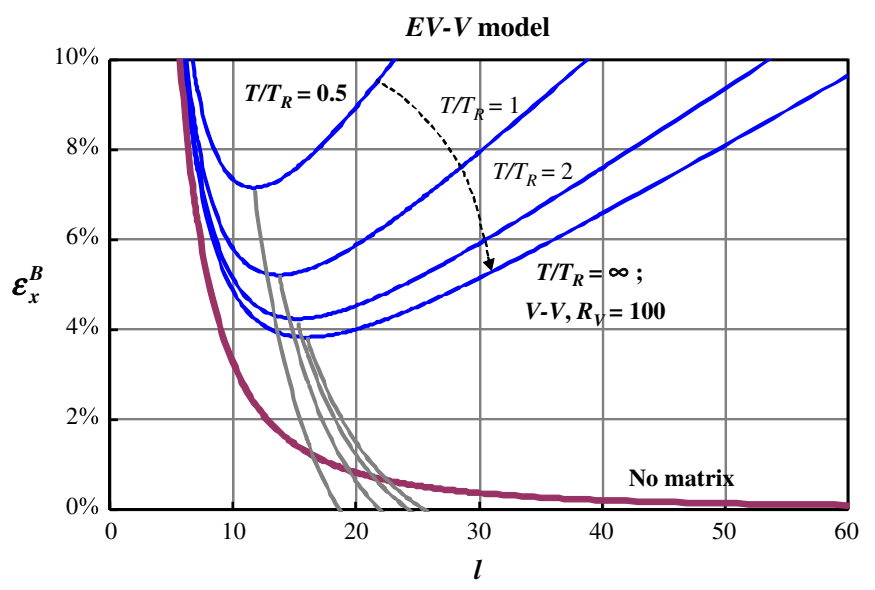

Fig. 10. The variation of the $\varepsilon_{x}^{B}-l$ curve with strain rates for the $E V-V$ model. There is a lower boundary ( $V-V$ model curve). A slower strain rate brings the $\varepsilon_{x}^{B}-l$ curve closer to the lower boundary. 


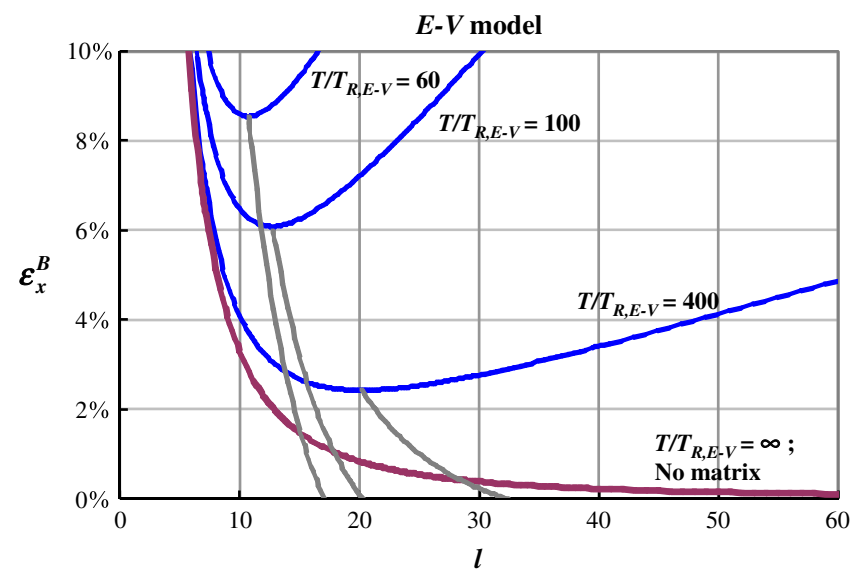

Fig. 11. The variation of the $\varepsilon_{x}^{B}-l$ curve with strain rates for the $E-V$ model. There is a lower boundary ("no matrix" curve). A slower strain rate brings the $\varepsilon_{x}^{B}-l$ curve closer to the lower boundary.

rate is extremely slow, the $\varepsilon_{x}^{B}-l$ curves of the $E-V$ system have a lower boundary, which is the "no matrix" curve, as shown in Fig. 11.

In fact, the $E-V$ system is a combinational extreme of the $E-E V$ and the $E V-V$ models: the $\varepsilon_{x}^{B}-l$ curves of the $E-V$ system does not have an upper boundary, just like the $E V-V$ model, and has a lower boundary, the "no matrix" curve, just like the $E-E V$ model.

For the $E-V$ model, the analytical solution proposed in this research is strain rate dependent, while the solutions proposed by Biot (1961) are rate independent. Looking into the derivation processes, both solutions are based on the same governing equation, which specifies the stress equilibrium of the layermatrix system along $y$-direction. The basic waveform used to find the solution of the governing equation and considered by Biot (1961) is $Y(x, t)=\mathrm{e}^{r t} \sin (\lambda x)$. As a result, Biot's proposed dominant wavelength is $l_{\mathrm{d}}=\pi \sqrt{\bar{E} / \sigma_{x}}$, in which only the stiffness $\bar{E}$ and the lateral stress $\sigma_{x}$ are involved and the viscosity of the matrix $\eta_{o}$ is not involved. Therefore, this solution suggests that the dominant wavelength of the layer will not be affected by viscous properties of the matrix, as if the matrix does not exist. It is difficult to perceive that the behavior of an elastic layer embedded in viscous matrix (the $E-V$ model) is exactly the same as the behavior of an elastic layer without being embedded in matrix.

On the other hand, the basic waveform adopted in this research is $Y(x, t)=A \varepsilon_{x}^{B}(t) \sin \lambda x$ and the dominant wavelength is $l_{\mathrm{d}}=2 \pi \sqrt[3]{\bar{G} T / 12 \eta_{o}}$, in which the viscosity of the matrix does have influence on the wavelength. When the layer is converted from one equilibrium state (shortened along $x$-direction) to another buckled equilibrium state (sinusoidal waveform), the displacement of the layer along $y$-direction can induce strain rate (or stress) within the matrix and, in turn, the matrix will exert vertical stress as $q=-4 \eta_{o} \lambda(\partial Y / \partial t)$, which is part of the governing equation. As such, the governing equation has already indicated that viscosity will affect the dominant wavelength.

The proposed solution also indicates that the resulted wavelength is rate dependent since the alternative measure of strain rate $T$ is involved. In the $V-V$ model, both the layer and the matrix are rate dependent and have the viscosity $\eta$ and $\eta_{o}$, respectively. The influence of $T$ does not show up since $T$ is eliminated in the term $R_{V}=(\eta / T) /\left(\eta_{o} / T\right)$ so that $R_{V}$ is the only factor controlling the dominant wavelength $\left(l_{\mathrm{d}}=\right.$ $\left.2 \pi \sqrt[3]{R_{V} / 6}\right)$. For the $E-V$ model, the layer does not have $\eta$ but has the rate independent elastic modulus. As a result, the term $T$ cannot be eliminated and the layer-matrix system is strain rate dependent. Overall, the proposed analytical solution better presents physical significance of the $E-V$ model.

\section{Conclusions}

The present research derived with analytical solutions that supplement existing niches and thus allow full solutions for the elastic, visco-elastic and viscous materials involved in fold buckling. The three basic types of fold-forms, Types A, $B$ and $C$, have been taken into consideration. Further research on these fold-forms, using the numerical method for instance, will justify the solutions proposed in the present study.

In this research, the $\varepsilon_{x}^{B}-l$ relationships revealed by the solutions were explored to unravel the nature and to give physical insights into buckling folds. The solutions are characterized by: (1) covering all three types of fold-forms; (2) involving elastic, visco-elastic and viscous materials; (3) proposing indices, including $T / T_{\mathrm{R}}$ and $I_{v}$, measuring strain rate; and (4) consistency under extreme conditions, including extreme strain rates or extreme types of materials. The influences of the strain rates and the competence contrasts $\left(R_{E}\right.$ and $\left.R_{V}\right)$ are demonstrated for each layer-matrix system.

This research faces limitations encountered in previous similar researches as follows.

1. The material models adopted are simplified. More sophisticated material models would be helpful in producing predictions closer to reality.

2. Folding in nature is three-dimensional, whereas the foldforms studied in this research are two-dimensional. Moreover, the governing equations rendering the solutions are actually one-dimensional. Hence, more studies are required to identify possible discrepancies induced by the reduction of dimensions.

3. The solutions only describe the fold-forms at the moment of folding initiation, but not the post-buckling behavior. We must keep in mind that the fold-forms found in the outcrops have undergone substantial post-buckle deformation. Caution should be exercised when comparing the results of the solution with the ones shown in outcrops. Methods have been proposed to restore outcrop post-buckle folds to their initial fold-forms (Schmalholz and Podladchikov, 2001a,b).

4. The range of competency contrast $(R)$ discussed in this work has the order of 100's, which is used for the purposes of highlighting the characteristics of the proposed solutions. The $R$ of natural rocks can be much lower than the ones discussed. Jeng et al. (2002) has discussed the possible range of $R$ corresponding to a given wavelength 
$l$ and found that if $l_{3}$ (wavelength of the Type $C$ fold-form) is also taken into consideration, the range of $R$ would be reasonable.

\section{Acknowledgements}

The supports from the National Science Council of Taiwan, NSC89-2211-E002-150 and NSC89-2211-E002-152, are acknowledged. The advices from reviewers, S.M. Schmalholz, B.E. Hobbs and T.G. Blenkinsop, have substantially enhanced the scientific soundness of this paper. We also thank Mr. Chris Fong in providing technical and linguistic advices.

\section{References}

Abbassi, M.R., Mancktelow, N.S., 1990. The effect of initial perturbation shape and symmetry on fold development. Journal of Structural Geology $12,273-282$

Abbassi, M.R., Mancktelow, N.S., 1992. Single layer buckle in non-linear material - I. Experimental study of fold development from an isolated initial perturbation. Journal of Structural Geology 14, 85-104.

Biot, M.A., 1957. Folding instability of a layered viscoelastic medium under compression. Proceedings of the Royal Society of London, Series A 242, 444-454.

Biot, M.A., 1959. Folding of a layered viscoelastic medium derived from an exact stability theory of a continuum under initial stress. Quarterly of Applied Mathematics 17, 185-204.

Biot, M.A., 1961. Theory of folding of stratified viscoelastic media and its implications in tectonics and orogenesis. Geological Society of America Bulletin 72, 1595-1620.

Budd, C.J., Peletier, M.A., 2000. Approximate self-similarity in models of geological folding. Society for Industrial and Applied Mathematics 60 (No. 3), 990-1016.

Cobbold, P.R., 1976. Fold shapes as functions of progressive strain. Philosophical Transactions of the Royal Society of London, Series A 283, 129-138.

Cobbold, P.R., 1977. Finite-element analysis of fold propagation - a problematic application? Tectonophysics 38, 339-353.

Currie, J.B., Patnode, H.W., Trump, R.P., 1962. Development of folds in sedimentary strata. Geological Society of America Bulletin 73, 655-674.

Donath, F.A., Parker, R.B., 1964. Folds and folding. Geological Society of America Bulletin 75, 45-62.

Dieterich, J.H., Carter, N.L., 1969. Stress-history of folding. American Journal of Science 267, 129-154.

Fletcher, R.C., 1974. Wavelength selection in the folding of a single layer with power-law rheology. American Journal of Science 274, 1029-1043.

Fletcher, R.C., 1977. Folding of a single viscous layer: exact infinitesimalamplitude solution. Tectonophysics 39, 593-606.

Hudleston, P.J., 1973. An analysis of "single-layer" folds developed experimentally in viscous media. Tectonophysics 16, 189-214.

Hudleston, P.J., Stephansson, O., 1973. Layer shortening and folding shape development in the buckling of single layers. Tectonophysics 17, 299321.

Hudleston, P.J., Lan, L., 1993. Rheological controls on the shapes of singlelayer folds. Journal of Structural Geology 16, 1007-1021.

Hunt, G.W., Mühlhaus, H.B., Whiting, A.I.M., 1996a. Evolution of localized folding for a thin elastic layer in a softening visco-elastic medium. Pure and Applied Geophysics 146, 229-252.

Hunt, G.W., Mühlhaus, H.B., Hobbs, B.E., Ord, A., 1996b. Localized folding of viscoelastic layers. Geologische Rundschau 85, 58-64.

Jeng, F.S., Lu, C.Y., Chang, N.G. 2001. Buckle folding of elastic strata. In: Proceedings of 10th International Conference on Computer Methods and Advances in Geomechanics, Tucson, USA, pp. 13091312 .
Jeng, F.S., Lai, Y.C., Teng, M.H., 2002. Influence of strain rate on buckle folding of an elasto-viscous single layer. Journal of Structural Geology 24, $501-516$.

Johnson, A.M., 1970. Physical Process in Geology. Freeman Cooper, San Francisco.

Johnson, A.M., 1977. Styles of Folding. Elsevier, New York.

Johnson, A.M., Fletcher, R.C., 1994. Folding of Viscous Layers. Columbia University Press, New York.

Karman, T.V., Biot, M.A., 1940. Mathematical Methods in Engineering. Mcgraw-Hill, New York.

Mancktelow, N.S., Abbassi, M.R., 1992. Single layer buckle folding in nonlinear materials - II comparison between theory and experiment. Journal of Structural Geology 14, 105-120.

Mancktelow, N.S., 1999. Finite-element modelling of single-layer folding in elasto-viscous materials: the effect of initial perturbation geometry. Journal of Structural Geology 21, 161-177.

Mühlhaus, H.-B., Hobbs, B.E., Ord, A., 1994. The role of axial constraints on the evolution of folds in single layers. In: Siriwardane, Zaman (Eds.), Computer Methods and Advances in Geomechanics. Balkema, Rotterdam, pp. 223-231.

Mühlhaus, H.B., Sakaguchi, H., Hobbs, B.E., 1998. Evolution of three-dimensional folds for a non-Newtonian plate in a viscous medium. Proceedings of the Royal Society of London, Series A 454, 1-23.

Price, N.J., Cosgrove, J.W., 1990. Analysis of Geological Structures. Cambridge University Press, Cambridge.

Ramberg, H., 1963. Fluid dynamics of viscous buckling applicable to folding of layered rocks. Bulletin of American Association of Petroleum Geologists $47,484-505$.

Ramberg, H., 1964. Selective buckling of composite layers with contrasted rheological properties, a theory for simultaneous formation of several orders of folds. Tectonophysics 1, 307-341.

Roberts, J.H., 1989. The Macmillan Field Guide to Geological Structures. The Macmillan Press Ltd., London.

Schmalholz, S.M., Podladchikov, Y.Y., 1999. Buckling versus folding: importance of viscoelasticity. Geophysical Research Letters 26 (17), 26412644.

Schmalholz, S.M., Podladchikov, Y.Y., 2000. Finite amplitude folding: transition from exponential to layer length controlled growth. Earth and Planetary Science Letters 179, 363-377.

Schmalholz, S.M., Podladchikov, Y.Y., 2001a. Strain and competence contrast estimation from fold shape. Tectonophysics 340, 195-213.

Schmalholz, S.M., Podladchikov, Y.Y., 2001b. A spectral/finite difference method for simulating large deformation of heterogeneous, visco-elastic materials. Geophysical Journal International 145, 199-208.

Schmalholz, S.M., Podladchikov, Y.Y., 2001c. Viscoelastic folding: Maxwell versus Kelvin rheology. Geophysical Research Letters 28 (9), 18351838.

Sherwin, J.A., Chapple, W.M., 1968. Wavelengths of single layer folds: a comparison between theory and observation. American Journal of Science 266, $167-179$.

Shimamoto, T., Hara, I., 1976. Geometry and strain distribution of single-layer folds. Tectonophysics 30, 1-34.

Smith, R.B., 1977. Formation of folds, boudinage and mullions in non-Newtonian materials. Geological Society of America Bulletin 88, 312-320.

Smoluchowski, M.S., 1909. Über ein gewisses Stabilitätsproblem der Elastizitätslehre und dessen Beziehung zur Entstehung von Faltengebirgen. Akad. Wissensch. Krakau, Math. Kl., 3-20.

Whiting, A.I.M., Hunt, G.W., 1997. Evolution of nonperiodic forms in geological folds. Mathematical Geology 29, 705-723.

Williams, J.R., Lewis, R.W., Zienkiewicz, O.C., 1977. A finite-element analysis of the role of initial perturbations in the folding of a single viscous layer. Tectonophysics 45, 187-200.

Zhang, Y., Hobbs, B.E., Ord, A., Mühlhaus, H.B., 1996. Computer simulation of single-layer buckling. Journal of Structural Geology 18, 643-655.

Zhang, Y., Mancktelow, N.S., Hobbs, B.E., Ord, A., Mühlhaus, H.B., 2000. Numerical modelling of single-layer folding: clarification of an issue regarding the possible effect of computer codes and the influence of initial irregularities. Journal of Structural Geology 22, 1511-1522. 